\title{
Algorithm of a new variational inclusion problem and strictly pseudononspreading mapping with application
}

Wongvisarut Khuangsatung and Atid Kangtunyakarn*

"Correspondence:

beawrock@hotmail.com

Department of Mathematics,

Faculty of Science, King Mongkut's

Institute of Technology Ladkrabang,

Bangkok 10520, Thailand

\begin{abstract}
The purpose of this research is to modify the variational inclusion problems and prove a strong convergence theorem for finding a common element of the set of fixed points of a $\kappa$-strictly pseudononspreading mapping and the set of solutions of a finite family of variational inclusion problems and the set of solutions of a finite family of equilibrium problems in Hilbert space. By using our main result, we prove a strong convergence theorem involving a $\kappa$-quasi-strictly pseudo-contractive mapping in Hilbert space. We give a numerical example to support some of our results.
\end{abstract}

Keywords: variational inclusion problems; $\kappa$-strictly pseudononspreading mapping; equilibrium problem; resolvent operator; fixed point problem

\section{Introduction}

Throughout this article, let $H$ be a real Hilbert space with inner product $\langle\cdot, \cdot\rangle$ and norm $\|\cdot\|$. Let $C$ be a nonempty closed convex subset of $H$. Let $\mathcal{S}: C \rightarrow C$ be a nonlinear mapping. A point $u \in C$ is called a fixed point of $\mathcal{S}$ if $\mathcal{S} u=u$. The set of fixed points of $\mathcal{S}$ is denoted by $\operatorname{Fix}(\mathcal{S}):=\{u \in C: \mathcal{S} u=u\}$. A mapping $\mathcal{S}$ is called nonexpansive if

$$
\|\mathcal{S} u-\mathcal{S} v\| \leq\|u-v\|, \quad \forall u, v \in C .
$$

In 2008, Kohsaka and Takahashi [1] introduced the nonspreading mapping in Hilbert space $H$ as follows:

$$
2\|\mathcal{S} u-\mathcal{S} v\|^{2} \leq\|\mathcal{S} u-v\|^{2}+\|u-\mathcal{S} v\|^{2}, \quad \forall u, v \in C .
$$

It is shown in [2] that (1.1) is equivalent to

$$
\|\mathcal{S} u-\mathcal{S} v\|^{2} \leq\|u-v\|^{2}+2\langle u-\mathcal{S} u, v-\mathcal{S} v\rangle, \quad \forall u, v \in C
$$

The mapping $\mathcal{S}: C \rightarrow C$ is called a $\kappa$-strictly pseudononspreading mapping if there exists $\kappa \in[0,1)$ such that

$$
\|\mathcal{S} u-\mathcal{S} v\|^{2} \leq\|u-v\|^{2}+\kappa\|(I-\mathcal{S}) u-(I-\mathcal{S}) v\|^{2}+2\langle u-\mathcal{S} u, v-\mathcal{S} v\rangle, \quad \forall u, v \in C .
$$


This mapping was introduced by Osilike and Isiogugu [3] in 2011. Clearly every nonspreading mapping is a $\kappa$-strictly pseudononspreading mapping.

Remark 1.1 Let $C$ be a nonempty closed convex subset of $H$. Then a mapping $\mathcal{S}: C \rightarrow C$ is a $\kappa$-strictly pseudononspreading if and only if

$$
\frac{1-\kappa}{2}\|(I-\mathcal{S}) u-(I-\mathcal{S}) v\|^{2} \leq\langle(I-\mathcal{S}) u-(I-\mathcal{S}) v, u-v\rangle+\langle(I-\mathcal{S}) u,(I-\mathcal{S}) v\rangle
$$

for all $u, v \in C$.

Proof Let $u, v \in C$ and $\mathcal{S}$ be a $\kappa$-strictly pseudononspreading mapping, then there exists $\kappa \in[0,1)$ such that

$$
\|\mathcal{S} u-\mathcal{S} v\|^{2} \leq\|u-v\|^{2}+\kappa\|(I-\mathcal{S}) u-(I-\mathcal{S}) v\|^{2}+2\langle u-\mathcal{S} u, v-\mathcal{S} v\rangle
$$

Since

$$
\|(I-\mathcal{S}) u-(I-\mathcal{S}) v\|^{2}=\|\mathcal{S} u-\mathcal{S} v\|^{2}-2\langle\mathcal{S} u-\mathcal{S} v, u-v\rangle+\|u-v\|^{2},
$$

then we have

$$
\|\mathcal{S} u-\mathcal{S} v\|^{2}=\|(I-\mathcal{S}) u-(I-\mathcal{S}) v\|^{2}+2\langle\mathcal{S} u-\mathcal{S} v, u-v\rangle-\|u-v\|^{2} .
$$

From (1.3) and (1.5), we have

$$
\begin{aligned}
& \|(I-\mathcal{S}) u-(I-\mathcal{S}) v\|^{2}+2\langle\mathcal{S} u-\mathcal{S} v, u-v\rangle-\|u-v\|^{2} \\
& \quad \leq\|u-v\|^{2}+\kappa\|(I-\mathcal{S}) u-(I-\mathcal{S}) v\|^{2}+2\langle u-\mathcal{S} u, v-\mathcal{S} v\rangle .
\end{aligned}
$$

It follows that

$$
\begin{aligned}
(1-\kappa)\|(I-\mathcal{S}) u-(I-\mathcal{S}) v\|^{2} & \leq 2\|u-v\|^{2}-2\langle\mathcal{S} u-\mathcal{S} v, u-v\rangle+2\langle u-\mathcal{S} u, v-\mathcal{S} v\rangle \\
& =2\langle(I-\mathcal{S}) u-(I-\mathcal{S}) v, u-v\rangle+2\langle u-\mathcal{S} u, v-\mathcal{S} v\rangle
\end{aligned}
$$

Then

$$
\frac{1-\kappa}{2}\|(I-\mathcal{S}) u-(I-\mathcal{S}) v\|^{2} \leq\langle(I-\mathcal{S}) u-(I-\mathcal{S}) v, u-v\rangle+\langle(I-\mathcal{S}) u,(I-\mathcal{S}) v\rangle
$$

On the other hand, let $u, v \in C$ and

$$
\begin{aligned}
\frac{1-\kappa}{2}\|(I-\mathcal{S}) u-(I-\mathcal{S}) v\|^{2} & \leq\langle(I-\mathcal{S}) u-(I-\mathcal{S}) v, u-v\rangle+\langle u-\mathcal{S} u, v-\mathcal{S} v\rangle \\
& =\|u-v\|^{2}-\langle\mathcal{S} u-\mathcal{S} v, u-v\rangle+\langle u-\mathcal{S} u, v-\mathcal{S} v\rangle .
\end{aligned}
$$

Then

$$
(1-\kappa)\|(I-\mathcal{S}) u-(I-\mathcal{S}) v\|^{2} \leq 2\|u-v\|^{2}-2\langle\mathcal{S} u-\mathcal{S} v, u-v\rangle+2\langle u-\mathcal{S} u, v-\mathcal{S} v\rangle .
$$


It follows that

$$
\begin{aligned}
2\langle\mathcal{S} u-\mathcal{S} u, u-v\rangle \leq & 2\|u-v\|^{2}-(1-\kappa)\|(I-\mathcal{S}) u-(I-\mathcal{S}) v\|^{2} \\
& +2\langle u-\mathcal{S} u, v-\mathcal{S} v\rangle
\end{aligned}
$$

From (1.4), we have

$$
2\langle\mathcal{S} u-\mathcal{S} v, u-v\rangle=\|\mathcal{S} u-\mathcal{S} v\|^{2}+\|u-v\|^{2}-\|(I-\mathcal{S}) u-(I-\mathcal{S}) v\|^{2}
$$

From (1.6) and (1.7), we have

$$
\begin{aligned}
& \|\mathcal{S} u-\mathcal{S} v\|^{2}+\|u-v\|^{2}-\|(I-\mathcal{S}) u-(I-\mathcal{S}) v\|^{2} \\
& \quad \leq 2\|u-v\|^{2}-(1-\kappa)\|(I-\mathcal{S}) u-(I-\mathcal{S}) v\|^{2}+2\langle u-\mathcal{S} u, v-\mathcal{S} v\rangle .
\end{aligned}
$$

Then

$$
\|\mathcal{S} u-\mathcal{S} v\|^{2} \leq\|u-v\|^{2}+\kappa\|(I-\mathcal{S}) u-(I-\mathcal{S}) v\|^{2}+2\langle(I-\mathcal{S}) u,(I-\mathcal{S}) v\rangle
$$

Example 1.2 Let $\mathcal{S}:[1, \infty) \rightarrow[1, \infty)$ be defined by

$$
\mathcal{S} u=\sin u, \quad \forall u \in[1, \infty)
$$

Then $\mathcal{S}$ is a $\kappa$-strictly pseudononspreading mapping where $\kappa \in[0,1)$.

Example 1.3 Let $\mathcal{S}:[0, \infty) \rightarrow[0, \infty)$ be defined by

$$
\mathcal{S} u=\frac{4 u^{2}}{5+4 u}, \quad \forall u \in C
$$

Then $\mathcal{S}$ is a $\frac{23}{25}$-strictly pseudononspreading mapping.

The mapping $A: C \rightarrow H$ is called $\alpha$-inverse strongly monotone if there exists a positive real number $\alpha$ such that

$$
\langle A u-A v, u-v\rangle \geq \alpha\|A u-A v\|^{2},
$$

for all $u, v \in C$.

Let $B: H \rightarrow H$ be a mapping and $M: H \rightarrow 2^{H}$ be a multi-valued mapping. The variational inclusion problem is to find $x \in H$ such that

$$
\theta \in B x+M x
$$

where $\theta$ is a zero vector in $H$. The set of the solution of $(1.8)$ is denoted by $\operatorname{VI}(H, B, M)$. It is well known that the variational inclusion problems are widely studied in mathematical programming, complementarity problems, variational inequalities, optimal control, mathematical economics, and game theory, etc. Many authors have increasingly investigated such a problem (1.8); see for instance [4-7] and references therein. 
Let $M: H \rightarrow 2^{H}$ be a multi-valued maximal monotone mapping, then the single-valued mapping $J_{M, \lambda}: H \rightarrow H$ defined by

$$
J_{M, \lambda}(x)=(I+\lambda M)^{-1}(x), \quad \forall x \in H,
$$

is called the resolvent operator associated with $M$, where $\lambda$ is any positive number and $I$ is an identity mapping; see [7].

Let $\Psi: C \times C \rightarrow \mathbb{R}$ be a bifunction. The equilibrium problem for $\Psi$ is to determine its equilibrium point. The set of solution of equilibrium problem is denoted by

$$
\operatorname{EP}(\Psi)=\{u \in C: \Psi(u, v) \geq 0, \forall v \in C\}
$$

Finding a solution of an equilibrium problem can be applied to many problems in physics, optimization, and economics. Many researchers have proposed some methods to solve the equilibrium problem; see, for example, $[8,9]$ and the references therein.

In 2008, Zhang et al. [7] introduced an iterative scheme for finding a common element of the set of solutions of the variational inclusion problem with multi-valued maximal monotone mapping and inverse strongly monotone mappings and the set of fixed points of nonexpansive mappings in Hilbert space as follows:

$$
\left\{\begin{array}{l}
v_{n}=J_{M, \lambda}\left(w_{n}-\lambda A w_{n}\right), \\
w_{n+1}=\alpha_{n} w+\left(1-\alpha_{n}\right) S v_{n}, \quad \forall n \geq 0,
\end{array}\right.
$$

and they proved a strong convergence theorem of the sequence $\left\{w_{n}\right\}$ under suitable conditions of the parameters $\left\{\alpha_{n}\right\}$ and $\lambda$.

In 2013, Kangtunyakarn [10] introduced an iterative algorithm for finding a common element of the set of fixed points of a $\kappa$-strictly pseudononspreading mapping and the set of solutions of a finite family of variational inequality problems as follows:

$$
w_{n+1}=\alpha_{n} u+\beta_{n} P_{C}\left(I-\lambda_{n}(I-\mathcal{S})\right) w_{n}+\gamma_{n} S w_{n}, \quad \forall n \in \mathbb{N}
$$

and proved a strong convergence theorem of the sequence $\left\{w_{n}\right\}$ under suitable conditions of the parameters $\left\{\alpha_{n}\right\},\left\{\beta_{n}\right\},\left\{\gamma_{n}\right\}$, and $\left\{\lambda_{n}\right\}$.

Very recently, Suwannaut and Kangtunyakarn [11] have modified (1.9) as follows:

$$
\operatorname{EP}\left(\sum_{i=1}^{N} a_{i} \Psi_{i}\right)=\left\{u \in C:\left(\sum_{i=1}^{N} a_{i} \Psi_{i}\right)(u, v) \geq 0, \forall v \in C\right\}
$$

where $\Psi_{i}: C \times C \rightarrow \mathbb{R}$ is for bifunctions and $a_{i}>0$ with $\sum_{i=1}^{N} a_{i}=1$ for every $i=1,2, \ldots, N$. It is obvious that (1.10) reduces to (1.9), if $\Psi_{i}=\Psi$, for all $i=1,2, \ldots, N$. They also introduced an iterative method for finding a common element of the set of fixed points of an infinite family of $\kappa_{i}$-strictly pseudo-contractive mappings and the set of solutions of a finite family of an equilibrium problem and a variational inequalities problem as follows:

$$
\left\{\begin{array}{l}
\sum_{i=1}^{N} a_{i} \Psi_{i}\left(z_{n}, y\right)+\frac{1}{r_{n}}\left\langle y-z_{n}, z_{n}-w_{n}\right\rangle \geq 0, \quad \forall y \in C, \\
w_{n+1}=\beta_{n}\left(\alpha_{n} \mu+\left(1-\alpha_{n}\right) S_{n} w_{n}\right)+\left(1-\beta_{n}\right) P_{C}\left(I-\rho_{n} \sum_{i=1}^{N} b_{i} A_{i}\right) z_{n}, \quad \forall n \geq 1 .
\end{array}\right.
$$


Under some appropriate conditions, they proved a strong convergence theorem of the sequence $\left\{w_{n}\right\}$ converging to an element of a set $\bigcap_{i=1}^{N} \operatorname{EP}\left(\Psi_{i}\right) \cap \bigcap_{i=1}^{N} \operatorname{VP}\left(A_{i}, C\right) \cap \bigcap_{i=1}^{\infty} \operatorname{Fix}\left(\mathcal{S}_{i}\right)$ where $A_{i}$ is a strongly positive linear bounded operator for every $i=1,2, \ldots, N$.

For $i=1,2, \ldots, N$, let $A_{i}: H \rightarrow H$ be a single-valued mapping and let $M: H \rightarrow 2^{H}$ be a multi-valued mapping. From the concept of (1.8), we introduce the problem of finding $u \in H$ such that

$$
\theta \in \sum_{i=1}^{N} a_{i} A_{i} u+M u,
$$

for all $a_{i} \in(0,1)$ with $\sum_{i=1}^{N} a_{i}=1$ and $\theta$ is a zero vector. This problem is called the modified variational inclusion. The set of solutions of (1.11) is denoted by $\mathrm{VI}\left(H, \sum_{i=1}^{N} a_{i} A_{i}, M\right)$. If $A_{i} \equiv A$ for all $i=1,2, \ldots, N$, then (1.11) reduces to (1.8).

In this paper, motivated by the research described above, we prove fixed point theory involving the modified variational inclusion and introduce iterative scheme for finding a common element of the set of fixed points of a $\kappa$-strictly pseudononspreading mapping and the set of solutions of a finite family of variational inclusion problems and the set of solutions of a finite family of equilibrium problem. By using the same method as our main theorem, we prove a strong convergence theorem for finding a common element of the set of fixed points of a $\kappa$-quasi-strictly pseudo-contractive mapping and the set of solutions of a finite family of variational inclusion problems and the set of solutions of a finite family of equilibrium problem in Hilbert space. Applying such a problem, we have a convergence theorem associated with a nonspreading mapping. In the last section, we also give numerical examples to support some of our results.

\section{Preliminaries}

In this paper, we denote weak and strong convergence by the notations ' - ' and ' $\rightarrow$ ', respectively. In a real Hilbert space $H$, recall that the (nearest point) projection $P_{C}$ from $H$ onto $C$ assigns to each $u \in H$ the unique point $P_{C} u \in C$ satisfying the property

$$
\left\|u-P_{C} v\right\|=\min _{v \in C}\|u-v\| .
$$

For a proof of the main theorem, we will use the following lemmas.

Lemma 2.1 ([12]) Given $u \in H$ and $v \in C$, then $P_{C} u=v$ if and only if we have the inequality

$$
\langle u-v, v-z\rangle \geq 0, \quad \forall z \in C .
$$

Lemma 2.2 ([13]) Let $\left\{p_{n}\right\}$ be a sequence of nonnegative real numbers satisfying

$$
p_{n+1} \leq\left(1-a_{n}\right) p_{n}+b_{n}, \quad \forall n \geq 0,
$$

where $\left\{a_{n}\right\}$ is a sequence in $(0,1)$ and $\left\{b_{n}\right\}$ is a sequence such that

(1) $\sum_{n=1}^{\infty} a_{n}=\infty$,

(2) $\lim \sup _{n \rightarrow \infty} \frac{b_{n}}{a_{n}} \leq 0$ or $\sum_{n=1}^{\infty}\left|b_{n}\right|<\infty$.

Then $\lim _{n \rightarrow \infty} p_{n}=0$. 
Lemma 2.3 Let $H$ be a real Hilbert space. Then

$$
\|u+v\|^{2} \leq\|u\|^{2}+2\langle v, u+v\rangle,
$$

for all $u, v \in H$.

Lemma 2.4 ([14]) Let $H$ be a Hilbert space. Then for all $u, v \in H$ and $\alpha_{i} \in[0,1]$ for $i=$ $1,2, \ldots, n$ such that $\alpha_{0}+\alpha_{1}+\cdots+\alpha_{n}=1$ the following equality holds:

$$
\left\|\alpha_{0} w_{0}+\alpha_{1} w_{1}+\cdots+\alpha_{n} w_{n}\right\|^{2}=\sum_{i=0}^{n} \alpha_{i}\left\|w_{i}\right\|^{2}-\sum_{0 \leq i, j \leq n} \alpha_{i} \alpha_{j}\left\|w_{i}-w_{j}\right\|^{2}
$$

For finding solutions of the equilibrium problem, assume a bifunction $\Psi: C \times C \rightarrow \mathbb{R}$ to satisfy the following conditions:

(A1) $\Psi(u, v)=0$ for all $u \in C$;

(A2) $\Psi$ is monotone, i.e., $\Psi(u, v)+\Psi(v, u) \leq 0$ for all $u, v \in C$;

(A3) for each $u, v, z \in C$,

$$
\lim _{t \downarrow 0} \Psi(t z+(1-t) u, v) \leq \Psi(u, v)
$$

(A4) for each $u \in C, v \mapsto \Psi(u, v)$ is convex and lower semicontinuous.

Lemma 2.5 ([15]) Let $C$ be a nonempty closed convex subset of $H$ and let $\Psi$ be a bifunction of $C \times C$ into $\mathbb{R}$ satisfying (A1)-(A4). Let $r>0$ and $u \in H$. Then there exists $z \in C$ such that

$$
\Psi(z, v)+\frac{1}{r}\langle v-z, z-u\rangle \geq 0, \quad \forall v \in C .
$$

Lemma 2.6 ([8]) Assume that $\Psi: C \times C \rightarrow \mathbb{R}$ satisfies (A1)-(A4). For $r>0$, define a mapping $\Theta_{r}: H \rightarrow C$ as follows:

$$
\Theta_{r}(u)=\left\{z \in C: \Psi(z, v)+\frac{1}{r}\langle v-z, z-u\rangle \geq 0, \forall v \in C\right\},
$$

for all $u \in H$. Then the following hold:

(i) $\Theta_{r}$ is single-valued;

(ii) $\Theta_{r}$ is firmly nonexpansive, i.e., for any $u, v \in H$,

$$
\left\|\Theta_{r}(u)-\Theta_{r}(v)\right\|^{2} \leq\left\langle\Theta_{r}(u)-\Theta_{r}(v), u-v\right\rangle ;
$$

(iii) $\operatorname{Fix}\left(\Theta_{r}\right)=\operatorname{EP}(\Psi)$;

(iv) $\mathrm{EP}(\Psi)$ is closed and convex.

Lemma 2.7 ([11]) Let $C$ be a nonempty closed convex subset of a real Hilbert space H. For $i=1,2, \ldots, N$, let $\Psi_{i}: C \times C \rightarrow \mathbb{R}$ be bifunctions satisfying (A1)-(A4) with $\bigcap_{i=1}^{N} \operatorname{EP}\left(\Psi_{i}\right) \neq \emptyset$. Then

$$
\operatorname{EP}\left(\sum_{i=1}^{N} a_{i} \Psi_{i}\right)=\bigcap_{i=1}^{N} \operatorname{EP}\left(\Psi_{i}\right),
$$

where $a_{i} \in(0,1)$ for every $i=1,2, \ldots, N$ and $\sum_{i=1}^{N} a_{i}=1$. 
Remark 2.8 ([11]) From Lemma 2.7,

$$
\operatorname{Fix}\left(\Theta_{r}\right)=\operatorname{EP}\left(\sum_{i=1}^{N} a_{i} \Psi_{i}\right)=\bigcap_{i=1}^{N} \operatorname{EP}\left(\Psi_{i}\right)
$$

where $a_{i} \in(0,1)$, for each $i=1,2, \ldots, N$, and $\sum_{i=1}^{N} a_{i}=1$.

Lemma 2.9 Let $C$ be a nonempty closed convex subset of a real Hilbert space $H$. Let $\sum_{i=1}^{N} a_{i} \Psi_{i}: C \times C \rightarrow \mathbb{R}$ be bifunctions satisfying (A1)-(A4) where $a_{i} \in(0,1)$, for each $i=1,2, \ldots, N$ and $\sum_{i=1}^{N} a_{i}=1$. For every $n \in \mathbb{N}$, let $0<c \leq r_{n} \leq d$ with $r_{n} \rightarrow r$ as $n \rightarrow \infty$. Then $\left\|\Theta_{r_{n}} u-\Theta_{r} u\right\| \rightarrow 0$ as $n \rightarrow \infty$ for all $u \in H$.

Proof For every $n \in \mathbb{N}$, let $0<c \leq r_{n} \leq d$ with $r_{n} \rightarrow r$ as $n \rightarrow \infty$, from which it follows that $0<c \leq r \leq d$. For every $u \in H$, by Lemma 2.6, we have

$$
\sum_{i=1}^{N} a_{i} \Psi_{i}\left(\Theta_{r_{n}} u, v\right)+\frac{1}{r_{n}}\left\langle v-\Theta_{r_{n}} u, \Theta_{r_{n}} u-u\right\rangle \geq 0, \quad \forall v \in C
$$

and

$$
\sum_{i=1}^{N} a_{i} \Psi_{i}\left(\Theta_{r} u, v\right)+\frac{1}{r}\left\langle v-\Theta_{r} u, \Theta_{r_{n}} u-u\right\rangle \geq 0, \quad \forall v \in C .
$$

In particular, we have

$$
\sum_{i=1}^{N} a_{i} \Psi_{i}\left(\Theta_{r_{n}} u, \Theta_{r} u\right)+\frac{1}{r_{n}}\left\langle\Theta_{r} u-\Theta_{r_{n}} u, \Theta_{r_{n}} u-u\right\rangle \geq 0
$$

and

$$
\sum_{i=1}^{N} a_{i} \Psi_{i}\left(\Theta_{r} u, \Theta_{r_{n}} u\right)+\frac{1}{r}\left\langle\Theta_{r_{n}} u-\Theta_{r} u, \Theta_{r} u-u\right\rangle \geq 0 .
$$

Summing up (2.1) and (2.2) and using (A2), we have

$$
\frac{1}{r}\left\langle\Theta_{r_{n}} u-\Theta_{r} u, \Theta_{r} u-u\right\rangle+\frac{1}{r_{n}}\left\langle\Theta_{r} u-\Theta_{r_{n}} u, \Theta_{r_{n}} u-u\right\rangle \geq 0 .
$$

It follows that

$$
\left\langle\Theta_{r_{n}} u-\Theta_{r} u, \frac{\Theta_{r} u-u}{r}-\frac{\Theta_{r_{n}} u-u}{r_{n}}\right\rangle \geq 0 .
$$

This implies that

$$
\begin{aligned}
0 & \leq\left\langle\Theta_{r} u-\Theta_{r_{n}} u, \Theta_{r_{n}} u-u-\frac{r_{n}}{r}\left(\Theta_{r} u-u\right)\right\rangle \\
& =\left\langle\Theta_{r} u-\Theta_{r_{n}} u, \Theta_{r_{n}} u-\Theta_{r} u+\left(1-\frac{r_{n}}{r}\right)\left(\Theta_{r} u-u\right)\right\rangle .
\end{aligned}
$$


It follows that

$$
\left\|\Theta_{r} u-\Theta_{r_{n}} u\right\|^{2} \leq\left|1-\frac{r_{n}}{r}\right|\left\|\Theta_{r} u-\Theta_{r_{n}} u\right\|\left(\left\|\Theta_{r} u\right\|+\|u\|\right) .
$$

Then we have

$$
\left\|\Theta_{r} u-\Theta_{r_{n}} u\right\| \leq \frac{1}{r}\left|r-r_{n}\right| L
$$

where $L=\sup \left\{\left\|\Theta_{r} u\right\|+\|u\|\right\}$. Since $r_{n} \rightarrow r$ as $n \rightarrow \infty$, we have

$$
\left\|\Theta_{r_{n}} u-\Theta_{r} u\right\| \rightarrow 0 \quad \text { as } n \rightarrow \infty .
$$

Remark 2.10 Let $\mathcal{S}: H \rightarrow H$ be a $\kappa$-strictly pseudononspreading mapping with $\operatorname{Fix}(\mathcal{S}) \neq \emptyset$. Define $T: H \rightarrow H$ by $T u:=((1-\lambda) I+\lambda \mathcal{S}) u$, where $\lambda \in(0,1-\kappa)$. Then the following hold:

(i) $\operatorname{Fix}(\mathcal{S})=\operatorname{Fix}(T)=\operatorname{Fix}(I-\lambda(I-\mathcal{S}))$;

(ii) for every $u \in H$ and $v \in \operatorname{Fix}(\mathcal{S})$,

$$
\|T u-v\| \leq\|u-v\| .
$$

Proof (i) It is easy to see that $\operatorname{Fix}(\mathcal{S})=\operatorname{Fix}(T)=\operatorname{Fix}(I-\lambda(I-\mathcal{S}))$.

(ii) Next, we show that $\|T u-v\| \leq\|u-v\|$. For every $u \in H$ and $v \in \operatorname{Fix}(\mathcal{S})$, we have

$$
\begin{aligned}
\|T u-v\|^{2}= & \|(I-\lambda(I-\mathcal{S})) u-v\|^{2} \\
= & \|(1-\lambda)(u-v)+\lambda(\mathcal{S} u-v)\|^{2} \\
= & (1-\lambda)\|u-v\|^{2}+\lambda\|\mathcal{S} u-v\|^{2}-\lambda(1-\lambda)\|\mathcal{S} u-u\|^{2} \\
\leq & (1-\lambda)\|u-v\|^{2}+\lambda\left(\|u-v\|^{2}+\kappa\|(I-\mathcal{S}) u\|^{2}\right) \\
& -\lambda(1-\lambda)\|\mathcal{S} u-u\|^{2} \\
= & \|u-v\|^{2}+\kappa \lambda\|\mathcal{S} u-u\|^{2}-\lambda(1-\lambda)\|\mathcal{S} u-u\|^{2} \\
= & \|u-v\|^{2}+\lambda(\lambda-(1-\kappa))\|\mathcal{S} u-u\|^{2} \\
\leq & \|u-v\|^{2} .
\end{aligned}
$$

Lemma 2.11 ([7]) $u \in H$ is a solution of variational inclusion (1.8) if and only if $u=J_{M, \lambda}(u-$ $\lambda B u), \forall \lambda>0$, i.e.,

$$
\mathrm{VI}(H, B, M)=\operatorname{Fix}\left(J_{M, \lambda}(I-\lambda B)\right), \quad \forall \lambda>0
$$

Further, if $\lambda \in(0,2 \alpha]$, then $\operatorname{VI}(H, B, M)$ is a closed convex subset in $H$.

Lemma 2.12 ([7]) The resolvent operator $J_{M, \lambda}$ associated with $M$ is single-valued, nonexpansive for all $\lambda>0$ and 1-inverse strongly monotone.

Lemma 2.13 Let $H$ be a real Hilbert space and let $M: H \rightarrow 2^{H}$ be a multi-valued maximal monotone mapping. For every $i=1,2, \ldots, N$, let $A_{i}: H \rightarrow H$ be $\alpha_{i}$-inverse strongly monotone 
mapping with $\eta=\min _{i=1,2, \ldots, N}\left\{\alpha_{i}\right\}$ and $\bigcap_{i=1}^{N} \operatorname{VI}\left(H, A_{i}, M\right) \neq \emptyset$. Then

$$
\mathrm{VI}\left(H, \sum_{i=1}^{N} a_{i} A_{i}, M\right)=\bigcap_{i=1}^{N} \mathrm{VI}\left(H, A_{i}, M\right),
$$

where $\sum_{i=1}^{N} a_{i}=1$, and $0<a_{i}<1$ for every $i=1,2, \ldots, N$. Moreover, $J_{M, \lambda}\left(I-\lambda \sum_{i=1}^{N} a_{i} A_{i}\right)$ is a nonexpansive mapping, for all $0<\lambda<2 \eta$.

Proof Clearly $\bigcap_{i=1}^{N} \mathrm{VI}\left(H, A_{i}, M\right) \subseteq \mathrm{VI}\left(H, \sum_{i=1}^{N} a_{i} A_{i}, M\right)$.

Let $u_{0} \in \mathrm{VI}\left(H, \sum_{i=1}^{N} a_{i} A_{i}, M\right)$ and let $u^{*} \in \bigcap_{i=1}^{N} \mathrm{VI}\left(H, A_{i}, M\right)$. From Lemma 2.11, we have

$$
u_{0} \in \operatorname{Fix}\left(J_{M, \lambda}\left(I-\lambda \sum_{i=1}^{N} a_{i} A_{i}\right)\right) .
$$

Since $\bigcap_{i=1}^{N} \operatorname{VI}\left(H, A_{i}, M\right) \subseteq \operatorname{VI}\left(H, \sum_{i=1}^{N} a_{i} A_{i}, M\right)$, we have $u^{*} \in \operatorname{VI}\left(H, \sum_{i=1}^{N} a_{i} A_{i}, M\right)$. From Lemma 2.11, we have

$$
u^{*} \in \operatorname{Fix}\left(J_{M, \lambda}\left(I-\lambda \sum_{i=1}^{N} a_{i} A_{i}\right)\right) .
$$

From the nonexpansiveness of $J_{M, \lambda}$, we have

$$
\begin{aligned}
\left\|u^{*}-u_{0}\right\|^{2} & =\left\|J_{M, \lambda}\left(I-\lambda \sum_{i=1}^{N} a_{i} A_{i}\right) u^{*}-J_{M, \lambda}\left(I-\lambda \sum_{i=1}^{N} a_{i} A_{i}\right) u_{0}\right\|^{2} \\
& \leq\left\|\left(I-\lambda \sum_{i=1}^{N} a_{i} A_{i}\right) u^{*}-\left(I-\lambda \sum_{i=1}^{N} a_{i} A_{i}\right) u_{0}\right\|^{2} \\
& =\left\|\left(u^{*}-u_{0}\right)-\lambda\left(\sum_{i=1}^{N} a_{i} A_{i} u^{*}-\sum_{i=1}^{N} a_{i} A_{i} u_{0}\right)\right\|^{2} \\
& \leq\left\|u^{*}-u_{0}\right\|^{2}-2 \lambda \sum_{i=1}^{N} a_{i}\left\langle u^{*}-u_{0}, A_{i} u^{*}-A_{i} u_{0}\right\rangle+\lambda^{2} \sum_{i=1}^{N} a_{i}\left\|A_{i} u^{*}-A_{i} u_{0}\right\|^{2} \\
& \leq\left\|u^{*}-u_{0}\right\|^{2}-2 \lambda \sum_{i=1}^{N} a_{i} \alpha_{i}\left\|A_{i} u^{*}-A_{i} u_{0}\right\|^{2}+\lambda^{2} \sum_{i=1}^{N} a_{i}\left\|A_{i} u^{*}-A_{i} u_{0}\right\|^{2} \\
& \leq\left\|u^{*}-u_{0}\right\|^{2}-2 \lambda \eta \sum_{i=1}^{N} a_{i}\left\|A_{i} u^{*}-A_{i} u_{0}\right\|^{2}+\lambda^{2} \sum_{i=1}^{N} a_{i}\left\|A_{i} u^{*}-A_{i} u_{0}\right\|^{2} \\
& =\left\|u^{*}-u_{0}\right\|^{2}+\lambda \sum_{i=1}^{N} a_{i}(\lambda-2 \eta)\left\|A_{i} u^{*}-A_{i} u_{0}\right\|^{2} \cdot
\end{aligned}
$$

This implies that

$$
\lambda \sum_{i=1}^{N} a_{i}(2 \eta-\lambda)\left\|A_{i} u^{*}-A_{i} u_{0}\right\|^{2} \leq 0 .
$$


Then

$$
A_{i} u^{*}=A_{i} u_{0}, \quad \forall i=1,2, \ldots, N
$$

Since $u_{0} \in \mathrm{VI}\left(H, \sum_{i=1}^{N} a_{i} A_{i}, M\right)$, we have

$$
\theta \in M u_{0}+\sum_{i=1}^{N} a_{i} A_{i} u_{0}
$$

From $u^{*} \in \mathrm{VI}\left(H, \sum_{i=1}^{N} a_{i} A_{i}, M\right)$, we have

$$
\theta \in M u^{*}+\sum_{i=1}^{N} a_{i} A_{i} u^{*}
$$

From (2.5) and (2.6), we have

$$
\theta \in M u_{0}+\sum_{i=1}^{N} a_{i} A_{i} u_{0}-M u^{*}-\sum_{i=1}^{N} a_{i} A_{i} u^{*} .
$$

From (2.4) and (2.7), we have

$$
\theta \in M u_{0}-M u^{*}
$$

Since $u^{*} \in \bigcap_{i=1}^{N} \operatorname{VI}\left(H, A_{i}, M\right)$ and we have (2.4) and (2.8),

$$
\theta \in M u_{0}-M u^{*}+M u^{*}+A_{i} u^{*}=M u_{0}+A_{i} u_{0},
$$

for all $i=1,2, \ldots, N$. It implies that $u_{0} \in \bigcap_{i=1}^{N} \operatorname{VI}\left(H, A_{i}, M\right)$.

Hence

$$
\mathrm{VI}\left(H, \sum_{i=1}^{N} a_{i} A_{i}, M\right) \subseteq \bigcap_{i=1}^{N} \mathrm{VI}\left(H, A_{i}, M\right) .
$$

Applying (2.3), we can conclude that $J_{M, \lambda}\left(I-\lambda \sum_{i=1}^{N} a_{i} A_{i}\right)$ is a nonexpansive mapping for all $i=1,2, \ldots, N$.

\section{Main result}

Theorem 3.1 Let $C$ be a nonempty closed convex subset of a real Hilbert space $H$. Let $M: H \rightarrow 2^{H}$ be a multi-valued maximal monotone mapping. For every $i=1,2, \ldots, N$, let $\Psi_{i}: C \times C \rightarrow \mathbb{R}$ be a bifunction satisfying (A1)-(A4) and $A_{i}: H \rightarrow H$ be $\alpha_{i}$-inverse strongly monotone mapping with $\eta=\min _{i=1,2, \ldots, N}\left\{\alpha_{i}\right\}$. Let $\mathcal{S}: H \rightarrow H$ be a $\kappa$-strictly pseudononspreading mapping. Assume $\Phi:=\operatorname{Fix}(\mathcal{S}) \cap \bigcap_{i=1}^{N} \operatorname{EP}\left(\Psi_{i}\right) \cap \bigcap_{i=1}^{N} \mathrm{VI}\left(H, A_{i}, M\right) \neq \emptyset$. Let the sequences $\left\{w_{n}\right\}$ and $\left\{z_{n}\right\}$ be generated by $w_{1}, \mu \in H$ and

$$
\left\{\begin{array}{c}
\sum_{i=1}^{N} a_{i} \Psi_{i}\left(z_{n}, y\right)+\frac{1}{r_{n}}\left\langle y-z_{n}, z_{n}-w_{n}\right\rangle \geq 0, \quad \forall y \in C, \\
w_{n+1}=\alpha_{n} \mu+\beta_{n} w_{n}+\gamma_{n} J_{M, \lambda}\left(I-\lambda \sum_{i=1}^{N} b_{i} A_{i}\right) w_{n} \\
+\eta_{n}\left(I-\rho_{n}(I-\mathcal{S})\right) w_{n}+\delta_{n} z_{n}, \quad \forall n \geq 1,
\end{array}\right.
$$


where $\left\{\alpha_{n}\right\},\left\{\beta_{n}\right\},\left\{\gamma_{n}\right\},\left\{\eta_{n}\right\},\left\{\delta_{n}\right\} \subseteq(0,1)$ and $\lambda>0$ with $\alpha_{n}+\beta_{n}+\gamma_{n}+\eta_{n}+\delta_{n}=1,0<\alpha<1$, and $0 \leq a_{i}, b_{i} \leq 1$, for every $i=1,2, \ldots, N, r_{n} \in[c, d] \subset(0,1), 0<p \leq \beta_{n}, \gamma_{n}, \eta_{n}, \delta_{n} \leq q<1$, $\rho_{n} \in(0,1-\kappa)$ for all $n \geq 1$. Suppose the following conditions hold:

(i) $\lim _{n \rightarrow \infty} \alpha_{n}=0$ and $\sum_{n=1}^{\infty} \alpha_{n}=\infty$,

(ii) $\sum_{n=1}^{\infty} \rho_{n}<\infty$,

(iii) $0<\lambda<2 \eta$, where $\eta=\min _{i=1,2, \ldots, N}\left\{\alpha_{i}\right\}$,

(iv) $\sum_{i=1}^{N} a_{i}=\sum_{i=1}^{N} b_{i}=1$,

(v) $\sum_{n=1}^{\infty}\left|\alpha_{n+1}-\alpha_{n}\right|<\infty, \sum_{n=1}^{\infty}\left|\beta_{n+1}-\beta_{n}\right|<\infty, \sum_{n=1}^{\infty}\left|\gamma_{n+1}-\gamma_{n}\right|<\infty$, $\sum_{n=1}^{\infty}\left|\rho_{n+1}-\rho_{n}\right|<\infty, \sum_{n=1}^{\infty}\left|\delta_{n+1}-\delta_{n}\right|<\infty, \sum_{n=1}^{\infty}\left|r_{n+1}-r_{n}\right|<\infty$.

Then the sequences $\left\{w_{n}\right\}$ and $\left\{z_{n}\right\}$ converge strongly to $\omega=P_{\Phi} \mu$.

Proof The proof of Theorem 3.1 will be divided into five steps:

Step 1 . We show that the sequence $\left\{w_{n}\right\}$ is bounded.

Since $\sum_{i=1}^{N} a_{i} \Psi_{i}$ satisfies (A1)-(A4), and

$$
\sum_{i=1}^{N} a_{i} \Psi_{i}\left(z_{n}, y\right)+\frac{1}{r_{n}}\left\langle y-z_{n}, z_{n}-w_{n}\right\rangle \geq 0, \quad \forall y \in C,
$$

by Lemma 2.6 and Remark 2.8, we have $z_{n}=\Theta_{r_{n}} w_{n}$ and $\operatorname{Fix}\left(\Theta_{r_{n}}\right)=\bigcap_{i=1}^{N} \operatorname{EP}\left(\Psi_{i}\right)$.

Let $\omega \in \Phi$. From Lemma 2.11 and Lemma 2.13, we have

$$
\omega=J_{M, \lambda}\left(I-\lambda \sum_{i=1}^{N} b_{i} A_{i}\right) \omega .
$$

From the nonexpansiveness of $J_{M, \lambda}\left(I-\lambda \sum_{i=1}^{N} b_{i} A_{i}\right)$, we have

$$
\left\|J_{M, \lambda}\left(I-\lambda \sum_{i=1}^{N} b_{i} A_{i}\right) w_{n}-\omega\right\| \leq\left\|w_{n}-\omega\right\| .
$$

From Remark 2.10, we have

$$
\begin{aligned}
\left\|\left(I-\rho_{n}(I-\mathcal{S})\right) w_{n}-\omega\right\|^{2} & =\left\|\left(1-\rho_{n}\right) w_{n}+\rho_{n} \mathcal{S} w_{n}-\omega\right\|^{2} \\
& \leq\left\|w_{n}-\omega\right\|^{2} .
\end{aligned}
$$

From the definition of $w_{n},(3.2)$, and (3.3), we have

$$
\begin{aligned}
\left\|w_{n+1}-\omega\right\|= & \| \alpha_{n} \mu+\beta_{n} w_{n}+\gamma_{n} J_{M, \lambda}\left(I-\lambda \sum_{i=1}^{N} b_{i} A_{i}\right) w_{n} \\
& +\eta_{n}\left(I-\rho_{n}(I-\mathcal{S})\right) w_{n}+\delta_{n} z_{n}-\omega \| \\
\leq & \alpha_{n}\|\mu-\omega\|+\beta_{n}\left\|w_{n}-\omega\right\|+\gamma_{n}\left\|J_{M, \lambda}\left(I-\lambda \sum_{i=1}^{N} b_{i} A_{i}\right) w_{n}-\omega\right\| \\
& +\eta_{n}\left\|\left(I-\rho_{n}(I-\mathcal{S})\right) w_{n}-\omega\right\|+\delta_{n}\left\|z_{n}-\omega\right\| \\
\leq & \alpha_{n}\|\mu-\omega\|+\left(1-\alpha_{n}\right)\left\|w_{n}-\omega\right\| \\
\leq & \max \left\{\|\mu-\omega\|,\left\|w_{1}-\omega\right\|\right\}=K .
\end{aligned}
$$


By mathematical induction, we have $\left\|w_{n}-z\right\| \leq K, \forall n \in \mathbb{N}$. It implies that $\left\{w_{n}\right\}$ is bounded and so is $\left\{z_{n}\right\}$.

By continuing in the same direction as in Step 1 of Theorem 3.1 in [10], we have

$$
\left\|\mathcal{S} w_{n}-\omega\right\| \leq \frac{1+\kappa}{1-\kappa}\left\|w_{n}-\omega\right\| .
$$

From (3.4), we can conclude that $\left\{\mathcal{S} w_{n}\right\}$ is bounded.

Step 2. Put $G=\sum_{i=1}^{N} b_{i} A_{i}$ and $P=I-\mathcal{S}$. We will show that $\lim _{n \rightarrow \infty}\left\|w_{n+1}-w_{n}\right\|=0$. From the definition of $w_{n}$, we have

$$
\begin{aligned}
\left\|w_{n+1}-w_{n}\right\|= & \| \alpha_{n} \mu+\beta_{n} w_{n}+\gamma_{n} J_{M, \lambda}(I-\lambda G) w_{n}+\eta_{n}\left(I-\rho_{n} P\right) w_{n} \\
& +\delta_{n} z_{n}-\alpha_{n-1} \mu-\beta_{n-1} w_{n-1}-\gamma_{n-1} J_{M, \lambda}(I-\lambda G) w_{n-1} \\
& -\eta_{n-1}\left(I-\rho_{n-1} P\right) w_{n-1}-\delta_{n-1} z_{n-1} \| \\
\leq & \left|\alpha_{n}-\alpha_{n-1}\right|\|\mu\|+\beta_{n}\left\|w_{n}-w_{n-1}\right\|+\left|\beta_{n}-\beta_{n-1}\right|\left\|w_{n-1}\right\| \\
& +\gamma_{n}\left\|J_{M, \lambda}(I-\lambda G) w_{n}-J_{M, \lambda}(I-\lambda G) w_{n-1}\right\| \\
& +\left|\gamma_{n}-\gamma_{n-1}\right|\left\|J_{M, \lambda}(I-\lambda G) w_{n-1}\right\| \\
& +\eta_{n}\left\|\left(I-\rho_{n} P\right) w_{n}-\left(I-\rho_{n-1} P\right) w_{n-1}\right\|+\left|\eta_{n}-\eta_{n-1}\right|\left\|\left(1-\rho_{n-1} P\right) w_{n-1}\right\| \\
& +\delta_{n}\left\|z_{n}-z_{n-1}\right\|+\left|\delta_{n}-\delta_{n-1}\right|\left\|z_{n-1}\right\| \\
\leq & \left|\alpha_{n}-\alpha_{n-1}\right|\|\mu\|+\beta_{n}\left\|w_{n}-w_{n-1}\right\|+\left|\beta_{n}-\beta_{n-1}\right|\left\|w_{n-1}\right\|+\gamma_{n}\left\|w_{n}-w_{n-1}\right\| \\
& +\left|\gamma_{n}-\gamma_{n-1}\right|\left\|J_{M, \lambda}(I-\lambda G) w_{n-1}\right\|+\left|\eta_{n}-\eta_{n-1}\right|\left\|\left(I-\rho_{n-1} P\right) w_{n-1}\right\| \\
& +\eta_{n}\left(\left\|w_{n}-w_{n-1}\right\|+\rho_{n}\left\|P w_{n}-P w_{n-1}\right\|+\left|\rho_{n}-\rho_{n-1}\right|\left\|P w_{n-1}\right\|\right) \\
& +\delta_{n}\left\|z_{n}-z_{n-1}\right\|+\left|\delta_{n}-\delta_{n-1}\right|\left\|z_{n-1}\right\| \\
\leq & \left|\alpha_{n}-\alpha_{n-1}\right|\|\mu\|+\beta_{n}\left\|w_{n}-w_{n-1}\right\|+\left|\beta_{n}-\beta_{n-1}\right|\left\|w_{n-1}\right\|+\gamma_{n}\left\|w_{n}-w_{n-1}\right\| \\
& +\left|\gamma_{n}-\gamma_{n-1}\right|\left\|J_{M, \lambda}(I-\lambda G) w_{n-1}\right\|+\left|\eta_{n}-\eta_{n-1}\right|\left\|\left(I-\rho_{n-1} P\right) w_{n-1}\right\| \\
& +\eta_{n}\left\|w_{n}-w_{n-1}\right\|+\rho_{n}\left\|P w_{n}-P w_{n-1}\right\|+\left|\rho_{n}-\rho_{n-1}\right|\left\|P w_{n-1}\right\| \\
& +\delta_{n}\left\|z_{n}-z_{n-1}\right\|+\left|\delta_{n}-\delta_{n-1}\right|\left\|z_{n-1}\right\| .
\end{aligned}
$$

By continuing in the same direction as in Step 2 of Theorem 3.1 in [11], we have

$$
\left\|z_{n}-z_{n-1}\right\| \leq\left\|w_{n}-w_{n-1}\right\|+\frac{1}{d}\left|r_{n}-r_{n-1}\right|\left\|z_{n}-w_{n}\right\| .
$$

By substituting (3.6) into (3.5), we obtain

$$
\begin{aligned}
\left\|w_{n+1}-w_{n}\right\| \leq & \left|\alpha_{n}-\alpha_{n-1}\right|\|\mu\|+\beta_{n}\left\|w_{n}-w_{n-1}\right\|+\left|\beta_{n}-\beta_{n-1}\right|\left\|w_{n-1}\right\|+\gamma_{n}\left\|w_{n}-w_{n-1}\right\| \\
& +\left|\gamma_{n}-\gamma_{n-1}\right|\left\|J_{M, \lambda}(I-\lambda G) w_{n-1}\right\|+\left|\eta_{n}-\eta_{n-1}\right|\left\|\left(I-\rho_{n-1} P\right) w_{n-1}\right\| \\
& +\eta_{n}\left\|w_{n}-w_{n-1}\right\|+\rho_{n}\left\|P w_{n}-P w_{n-1}\right\|+\left|\rho_{n}-\rho_{n-1}\right|\left\|P w_{n-1}\right\| \\
& +\delta_{n}\left\|z_{n}-z_{n-1}\right\|+\left|\delta_{n}-\delta_{n-1}\right|\left\|z_{n-1}\right\| \\
\leq & \left|\alpha_{n}-\alpha_{n-1}\right|\|\mu\|+\beta_{n}\left\|w_{n}-w_{n-1}\right\|+\left|\beta_{n}-\beta_{n-1}\right|\left\|w_{n-1}\right\|+\gamma_{n}\left\|w_{n}-w_{n-1}\right\|
\end{aligned}
$$




$$
\begin{aligned}
& +\left|\gamma_{n}-\gamma_{n-1}\right|\left\|J_{M, \lambda}(I-\lambda G) w_{n-1}\right\|+\left|\eta_{n}-\eta_{n-1}\right|\left\|\left(I-\rho_{n-1} P\right) w_{n-1}\right\| \\
& +\eta_{n}\left\|w_{n}-w_{n-1}\right\|+\rho_{n}\left\|P w_{n}-P w_{n-1}\right\|+\left|\rho_{n}-\rho_{n-1}\right|\left\|P w_{n-1}\right\| \\
& +\delta_{n}\left(\left\|w_{n}-w_{n-1}\right\|+\frac{1}{d}\left|r_{n}-r_{n-1}\right|\left\|z_{n}-w_{n}\right\|\right)+\left|\delta_{n}-\delta_{n-1}\right|\left\|z_{n-1}\right\| \\
\leq & \left|\alpha_{n}-\alpha_{n-1}\right|\|\mu\|+\left(1-\alpha_{n}\right)\left\|w_{n}-w_{n-1}\right\|+\left|\beta_{n}-\beta_{n-1}\right|\left\|w_{n-1}\right\| \\
& +\left|\gamma_{n}-\gamma_{n-1}\right|\left\|J_{M, \lambda}(I-\lambda G) w_{n-1}\right\|+\left|\eta_{n}-\eta_{n-1}\right|\left\|\left(I-\rho_{n-1} P\right) w_{n-1}\right\| \\
& +\rho_{n}\left\|P w_{n}-P w_{n-1}\right\|+\left|\rho_{n}-\rho_{n-1}\right|\left\|P w_{n-1}\right\| \\
& +\frac{1}{d}\left|r_{n}-r_{n-1}\right|\left\|z_{n}-w_{n}\right\|+\left|\delta_{n}-\delta_{n-1}\right|\left\|z_{n-1}\right\| .
\end{aligned}
$$

Applying Lemma 2.2, (3.7), and the conditions (i), (ii), (v), we have

$$
\lim _{n \rightarrow \infty}\left\|w_{n+1}-w_{n}\right\|=0
$$

Step 3. We show that $\lim _{n \rightarrow \infty}\left\|z_{n}-w_{n}\right\|=\lim _{n \rightarrow \infty}\left\|\left(I-\rho_{n} P\right) w_{n}-w_{n}\right\|=\lim _{n \rightarrow \infty} \| J_{M, \lambda}(I-$ $\lambda G) w_{n}-w_{n} \|=0$. By the definition of $w_{n},(3.2)$, and (3.3), we have

$$
\begin{aligned}
\left\|w_{n+1}-\omega\right\|^{2}= & \| \alpha_{n} \mu+\beta_{n} w_{n}+\gamma_{n} J_{M, \lambda}(I-\lambda G) w_{n} \\
& +\eta_{n}\left(I-\rho_{n} P\right) w_{n}+\delta_{n} z_{n}-\omega \|^{2} \\
\leq & \alpha_{n}\|\mu-\omega\|^{2}+\beta_{n}\left\|w_{n}-\omega\right\|^{2}+\gamma_{n}\left\|J_{M, \lambda}(I-\lambda G) w_{n}-\omega\right\|^{2} \\
& +\eta_{n}\left\|\left(I-\rho_{n} P\right) w_{n}-\omega\right\|^{2}+\delta_{n}\left\|z_{n}-\omega\right\|^{2}-\beta_{n} \delta_{n}\left\|w_{n}-z_{n}\right\|^{2} \\
& -\beta_{n} \gamma_{n}\left\|J_{M, \lambda}(I-\lambda G) w_{n}-w_{n}\right\|^{2} \\
= & \alpha_{n}\|\mu-\omega\|^{2}+\left(1-\alpha_{n}\right)\left\|w_{n}-\omega\right\|^{2}-\beta_{n} \delta_{n}\left\|w_{n}-z_{n}\right\|^{2} \\
& -\beta_{n} \gamma_{n}\left\|J_{M, \lambda}(I-\lambda G) w_{n}-w_{n}\right\|^{2} \\
\leq & \alpha_{n}\|\mu-\omega\|^{2}+\left\|w_{n}-\omega\right\|^{2}-\beta_{n} \delta_{n}\left\|w_{n}-\omega_{n}\right\|^{2} \\
& \quad-\beta_{n} \gamma_{n}\left\|J_{M, \lambda}(I-\lambda G) w_{n}-w_{n}\right\|^{2} .
\end{aligned}
$$

It implies that

$$
\begin{aligned}
\beta_{n} \delta_{n}\left\|z_{n}-w_{n}\right\|^{2} \leq & \alpha_{n}\|\mu-\omega\|^{2}+\left\|w_{n}-\omega\right\|^{2}-\left\|w_{n+1}-\omega\right\|^{2} \\
& -\beta_{n} \gamma_{n}\left\|J_{M, \lambda}(I-\lambda G) w_{n}-w_{n}\right\|^{2} \\
\leq & \alpha_{n}\|\mu-\omega\|^{2}+\left(\left\|w_{n}-\omega\right\|+\left\|w_{n+1}-\omega\right\|\right)\left\|w_{n+1}-w_{n}\right\| .
\end{aligned}
$$

From the condition (i) and (3.8), we have

$$
\lim _{n \rightarrow \infty}\left\|z_{n}-w_{n}\right\|=0
$$

By continuing in the same direction as (3.9), we have

$$
\lim _{n \rightarrow \infty}\left\|J_{M, \lambda}(I-\lambda G) w_{n}-w_{n}\right\|=0 .
$$


From the definition of $w_{n}$, we have

$$
\begin{aligned}
w_{n+1}-w_{n}= & \alpha_{n}\left(\mu-w_{n}\right)+\gamma_{n}\left(J_{M, \lambda}(I-\lambda G) w_{n}-w_{n}\right) \\
& +\eta_{n}\left(\left(I-\rho_{n} P\right) w_{n}-w_{n}\right)+\delta_{n}\left(z_{n}-w_{n}\right) .
\end{aligned}
$$

From the condition (i), (3.8), (3.9), and (3.10), we have

$$
\lim _{n \rightarrow \infty}\left\|\left(I-\rho_{n}(I-\mathcal{S})\right) w_{n}-w_{n}\right\|=0
$$

Step 4. We will show that $\lim \sup _{n \rightarrow \infty}\left\langle\mu-\omega, w_{n}-\omega\right\rangle \leq 0$, where $\omega=P_{\Phi} \mu$.

To show this, choose a subsequence $\left\{w_{n_{k}}\right\}$ of $\left\{w_{n}\right\}$ such that

$$
\limsup _{n \rightarrow \infty}\left\langle\mu-\omega, w_{n}-\omega\right\rangle=\lim _{k \rightarrow \infty}\left\langle\mu-\omega, w_{n_{k}}-\omega\right\rangle
$$

Without loss of generality, we can assume that $w_{n_{k}} \rightarrow \xi$ as $k \rightarrow \infty$. From (3.9), we obtain $z_{n_{k}} \rightarrow \xi$ as $k \rightarrow \infty$.

First, we will show that $\xi \in \bigcap_{i=1}^{N} \operatorname{VI}\left(H, A_{i}, M\right)$. Assume that $\xi \notin \bigcap_{i=1}^{N} \operatorname{VI}\left(H, A_{i}, M\right)$. By Lemmas 2.11 and 2.13, $\bigcap_{i=1}^{N} \operatorname{VI}\left(H, A_{i}, M\right)=\operatorname{Fix}\left(J_{M, \lambda}((I-\lambda G))\right)$. Then $\xi \neq J_{M, \lambda}(I-\lambda G) \xi$, where $G=\sum_{i=1}^{N} b_{i} A_{i}$. By the nonexpansiveness of $J_{M, \lambda}((I-\lambda G))$, (3.10), and Opial's condition, we obtain

$$
\begin{aligned}
\lim _{k \rightarrow \infty} \inf \left\|w_{n_{k}}-\xi\right\|< & \lim _{k \rightarrow \infty} \inf \left\|w_{n_{k}}-J_{M, \lambda}((I-\lambda G)) \xi\right\| \\
\leq & \lim _{k \rightarrow \infty} \inf \left(\left\|w_{n_{k}}-J_{M, \lambda}((I-\lambda G)) w_{n_{k}}\right\|\right. \\
& \left.\quad+\left\|J_{M, \lambda}((I-\lambda G)) w_{n_{k}}-J_{M, \lambda}((I-\lambda G)) \xi\right\|\right) \\
\leq & \lim _{k \rightarrow \infty} \inf \left\|w_{n_{k}}-\xi\right\| .
\end{aligned}
$$

This is a contradiction. Then we have

$$
\xi \in \bigcap_{i=1}^{N} \operatorname{VI}\left(H, A_{i}, M\right) .
$$

Next, we will show that $\xi \in \operatorname{Fix}(\mathcal{S})$. Assume that $\xi \notin \operatorname{Fix}(\mathcal{S})$. From Remark 2.10(i), we get $\operatorname{Fix}(\mathcal{S})=\operatorname{Fix}\left(I-\rho_{n_{k}}(I-\mathcal{S})\right)$. Then $\xi \neq\left(I-\rho_{n_{k}}(I-\mathcal{S})\right) \xi$. From the condition (ii), (3.11), and Opial's condition, we obtain

$$
\begin{aligned}
\lim _{k \rightarrow \infty} \inf \left\|w_{n_{k}}-\xi\right\|< & \lim _{k \rightarrow \infty} \inf \left\|w_{n_{k}}-\left(I-\rho_{n_{k}}(I-\mathcal{S})\right) \xi\right\| \\
\leq & \lim _{k \rightarrow \infty} \inf \left(\left\|w_{n_{k}}-\left(I-\rho_{n_{k}}(I-\mathcal{S})\right) w_{n_{k}}\right\|\right. \\
& \left.+\left\|\left(I-\rho_{n_{k}}(I-\mathcal{S})\right) w_{n_{k}}-\left(I-\rho_{n_{k}}(I-\mathcal{S})\right) \xi\right\|\right) \\
\leq & \lim _{k \rightarrow \infty} \inf \left(\left\|w_{n_{k}}-\left(I-\rho_{n_{k}}(I-\mathcal{S})\right) w_{n_{k}}\right\|\right. \\
& \left.+\left\|w_{n_{k}}-\xi\right\|+\rho_{n_{k}}\left\|(I-\mathcal{S}) w_{n_{k}}-(I-\mathcal{S}) \xi\right\|\right) \\
= & \lim _{k \rightarrow \infty} \inf \left\|w_{n_{k}}-\xi\right\| .
\end{aligned}
$$


This is a contradiction. Then we have

$$
\xi \in \operatorname{Fix}(\mathcal{S}) .
$$

Since $0<c \leq r_{n} \leq d, \forall n \in \mathbb{N}$, then we have $r_{n_{k}} \rightarrow r$ as $k \rightarrow \infty$ with $0<c \leq r \leq d$. Applying Lemma 2.9, we have $\left\|\Theta_{r_{n_{k}}} w_{n_{k}}-\Theta_{r} w_{n_{k}}\right\| \rightarrow 0$ as $k \rightarrow \infty$. Next, we will show that $\xi \in \bigcap_{i=1}^{N} \operatorname{EP}\left(\Psi_{i}\right)$. Assume that $\xi \notin \bigcap_{i=1}^{N} \operatorname{EP}\left(\Psi_{i}\right)$. From Remark 2.8, we have $\xi \notin \operatorname{Fix}\left(\Theta_{r}\right)$. By Opial's condition and (3.9), we have

$$
\begin{aligned}
\lim _{k \rightarrow \infty} \inf \left\|w_{n_{k}}-\xi\right\|< & \lim _{k \rightarrow \infty} \inf \left\|w_{n_{k}}-\Theta_{r} \xi\right\| \\
\leq & \lim _{k \rightarrow \infty} \inf \left(\left\|w_{n_{k}}-\Theta_{r_{n_{k}}} w_{n_{k}}\right\|\right. \\
& \left.+\left\|\Theta_{r_{n_{k}}} w_{n_{k}}-\Theta_{r} w_{n_{k}}\right\|+\left\|\Theta_{r} w_{n_{k}}-\Theta_{r} \xi\right\|\right) \\
\leq & \lim _{k \rightarrow \infty} \inf \left\|w_{n_{k}}-\xi\right\| .
\end{aligned}
$$

This is a contradiction. Then we have

$$
\xi \in \bigcap_{i=1}^{N} \operatorname{EP}\left(\Psi_{i}\right) .
$$

From (3.13), (3.14), and (3.15), we can conclude that $\xi \in \Phi$.

Since $w_{n_{k}} \rightarrow \xi$ as $k \rightarrow \infty$ and $\xi \in \Phi$. By (3.12) and Lemma 2.1, we have

$$
\begin{aligned}
\limsup _{n \rightarrow \infty}\left\langle\mu-\omega, w_{n}-\omega\right\rangle & =\lim _{k \rightarrow \infty}\left\langle\mu-\omega, w_{n_{k}}-\omega\right\rangle \\
& =\langle\mu-\omega, \xi-\omega\rangle \\
& \leq 0 .
\end{aligned}
$$

Step 5. Finally, we will show that $\lim _{n \rightarrow \infty} w_{n}=\omega$, where $\omega=P_{\Phi} \mu$. From the definition of $w_{n}$, we have

$$
\begin{aligned}
\left\|w_{n+1}-\omega\right\|^{2}= & \left\|\alpha_{n} \mu+\beta_{n} w_{n}+\gamma_{n} J_{M, \lambda}(I-\lambda G) w_{n}+\eta_{n}\left(I-\rho_{n} P\right) w_{n}+\delta_{n} z_{n}-\omega\right\|^{2} \\
\leq & \| \alpha_{n}(\mu-\omega)+\beta_{n}\left(w_{n}-\omega\right)+\gamma_{n}\left(J_{M, \lambda}(I-\lambda G) w_{n}-\omega\right) \\
& +\eta_{n}\left(\left(I-\rho_{n} P\right) w_{n}-\omega\right)+\delta_{n}\left(z_{n}-\omega\right) \|^{2} \\
\leq & \left(\beta_{n}\left\|w_{n}-\omega\right\|+\gamma_{n}\left\|J_{M, \lambda}(I-\lambda G) w_{n}-\omega\right\|+\eta_{n}\left\|\left(I-\rho_{n} P\right) w_{n}-\omega\right\|\right. \\
& \left.+\delta_{n}\left\|z_{n}-\omega\right\|\right)^{2}+2 \alpha_{n}\left\langle\mu-\omega, w_{n+1}-\omega\right\rangle \\
\leq & \left(1-\alpha_{n}\right)\left\|w_{n}-\omega\right\|^{2}+2 \alpha_{n}\left\langle\mu-\omega, w_{n+1}-\omega\right\rangle .
\end{aligned}
$$

From the condition (i), (3.16), and Lemma 2.2, we can conclude that the sequence $\left\{w_{n}\right\}$ converges strongly to $\omega=P_{\Phi} \mu$. By (3.9), we find that $\left\{z_{n}\right\}$ converges strongly to $\omega=P_{\Phi} \mu$. This completes the proof.

As a direct proof of Theorem 3.1, we obtain the following results.

Corollary 3.2 Let $C$ be a nonempty closed convex subset of a real Hilbert space $H$ and let $M: H \rightarrow 2^{H}$ be a multi-valued maximal monotone mapping. For every $i=1,2, \ldots, N$, let 
$\Psi_{i}: C \times C \rightarrow \mathbb{R}$ be a bifunction satisfying (A1)-(A4) and let $A: H \rightarrow H$ be an $\alpha$-inverse strongly monotone mapping. Let $\mathcal{S}: H \rightarrow H$ be a $\kappa$-strictly pseudononspreading mapping. Assume $\Phi:=\operatorname{Fix}(\mathcal{S}) \cap \bigcap_{i=1}^{N} \operatorname{EP}\left(\Psi_{i}\right) \cap \operatorname{VI}(H, A, M) \neq \emptyset$. Let the sequences $\left\{w_{n}\right\}$ and $\left\{z_{n}\right\}$ be generated by $w_{1}, \mu \in H$ and

$$
\left\{\begin{array}{c}
\sum_{i=1}^{N} a_{i} \Psi_{i}\left(z_{n}, y\right)+\frac{1}{r_{n}}\left(y-z_{n}, z_{n}-w_{n}\right\rangle \geq 0, \quad \forall y \in C, \\
w_{n+1}=\alpha_{n} \mu+\beta_{n} w_{n}+\gamma_{n} J_{M, \lambda}(I-\lambda A) w_{n} \\
\quad+\eta_{n}\left(I-\rho_{n}(I-\mathcal{S})\right) w_{n}+\delta_{n} z_{n}, \quad \forall n \geq 1,
\end{array}\right.
$$

where $\left\{\alpha_{n}\right\},\left\{\beta_{n}\right\},\left\{\gamma_{n}\right\},\left\{\eta_{n}\right\},\left\{\delta_{n}\right\} \subseteq(0,1)$ and $\lambda>0$ with $\alpha_{n}+\beta_{n}+\gamma_{n}+\eta_{n}+\delta_{n}=1,0<\alpha<1$, and $0 \leq a_{i} \leq 1$, for every $i=1,2, \ldots, N, r_{n} \in[c, d] \subset(0,1), 0<p \leq \beta_{n}, \gamma_{n}, \eta_{n}, \delta_{n} \leq q<1$, $\rho_{n} \in(0,1-\kappa)$ for all $n \geq 1$. Suppose the following conditions hold:

(i) $\lim _{n \rightarrow \infty} \alpha_{n}=0$ and $\sum_{n=1}^{\infty} \alpha_{n}=\infty$,

(ii) $\sum_{n=1}^{\infty} \rho_{n}<\infty$,

(iii) $0<\lambda<2 \alpha$,

(iv) $\sum_{i=1}^{N} a_{i}=1$,

(v) $\sum_{n=1}^{\infty}\left|\alpha_{n+1}-\alpha_{n}\right|<\infty, \sum_{n=1}^{\infty}\left|\beta_{n+1}-\beta_{n}\right|<\infty, \sum_{n=1}^{\infty}\left|\gamma_{n+1}-\gamma_{n}\right|<\infty$, $\sum_{n=1}^{\infty}\left|\rho_{n+1}-\rho_{n}\right|<\infty, \sum_{n=1}^{\infty}\left|\delta_{n+1}-\delta_{n}\right|<\infty, \sum_{n=1}^{\infty}\left|r_{n+1}-r_{n}\right|<\infty$.

Then the sequences $\left\{w_{n}\right\}$ and $\left\{z_{n}\right\}$ converge strongly to $\omega=P_{\Phi} \mu$.

Proof Put $A_{i} \equiv A$ for all $i=1,2, \ldots, N$ in Theorem 3.1. So, from Theorem 3.1, we obtain the desired result.

Corollary 3.3 Let $C$ be a nonempty closed convex subset of a real Hilbert space $H$ and let $M: H \rightarrow 2^{H}$ be a multi-valued maximal monotone mapping. Let $\Psi: C \times C \rightarrow \mathbb{R}$ be a bifunction satisfying (A1)-(A4). For every $i=1,2, \ldots, N, A_{i}: H \rightarrow H$ be $\alpha_{i}$-inverse strongly monotone mapping with $\eta=\min _{i=1,2, \ldots, N}\left\{\alpha_{i}\right\}$. Let $\mathcal{S}: H \rightarrow H$ be a $\kappa$-strictly pseudononspreading mapping. Assume $\Phi:=\operatorname{Fix}(\mathcal{S}) \cap \mathrm{EP}(F) \cap \bigcap_{i=1}^{N} \mathrm{VI}\left(H, A_{i}, M\right) \neq \emptyset$. Let the sequences $\left\{w_{n}\right\}$ and $\left\{z_{n}\right\}$ be generated by $w_{1}, \mu \in H$ and

$$
\left\{\begin{array}{c}
\Psi\left(z_{n}, y\right)+\frac{1}{r_{n}}\left\langle y-z_{n}, z_{n}-w_{n}\right\rangle \geq 0, \quad \forall y \in C, \\
w_{n+1}=\alpha_{n} \mu+\beta_{n} w_{n}+\gamma_{n} J_{M, \lambda}\left(I-\lambda \sum_{i=1}^{N} b_{i} A_{i}\right) w_{n} \\
+\eta_{n}\left(I-\rho_{n}(I-\mathcal{S})\right) w_{n}+\delta_{n} z_{n}, \quad \forall n \geq 1,
\end{array}\right.
$$

where $\left\{\alpha_{n}\right\},\left\{\beta_{n}\right\},\left\{\gamma_{n}\right\},\left\{\eta_{n}\right\},\left\{\delta_{n}\right\} \subseteq(0,1)$ and $\lambda>0$ with $\alpha_{n}+\beta_{n}+\gamma_{n}+\eta_{n}+\delta_{n}=1,0<\alpha<1$, and $0 \leq b_{i} \leq 1$, for every $i=1,2, \ldots, N, r_{n} \in[c, d] \subset(0,1), 0<p \leq \beta_{n}, \gamma_{n}, \eta_{n}, \delta_{n} \leq q<1$, $\rho_{n} \in(0,1-\kappa)$ for all $n \geq 1$. Suppose the following conditions hold:

(i) $\lim _{n \rightarrow \infty} \alpha_{n}=0$ and $\sum_{n=1}^{\infty} \alpha_{n}=\infty$,

(ii) $\sum_{n=1}^{\infty} \rho_{n}<\infty$,

(iii) $0<\lambda<2 \eta$, where $\eta=\min _{i=1,2,3, \ldots, N}\left\{\alpha_{i}\right\}$,

(iv) $\sum_{i=1}^{N} b_{i}=1$,

(v) $\sum_{n=1}^{\infty}\left|\alpha_{n+1}-\alpha_{n}\right|<\infty, \sum_{n=1}^{\infty}\left|\beta_{n+1}-\beta_{n}\right|<\infty, \sum_{n=1}^{\infty}\left|\gamma_{n+1}-\gamma_{n}\right|<\infty$, $\sum_{n=1}^{\infty}\left|\rho_{n+1}-\rho_{n}\right|<\infty, \sum_{n=1}^{\infty}\left|\delta_{n+1}-\delta_{n}\right|<\infty, \sum_{n=1}^{\infty}\left|r_{n+1}-r_{n}\right|<\infty$.

Then the sequences $\left\{w_{n}\right\}$ and $\left\{z_{n}\right\}$ converge strongly to $\omega=P_{\Phi} \mu$.

Proof Take $\Psi=\Psi_{i}, \forall i=1,2, \ldots, N$. By Theorem 3.1, we obtain the desired conclusion. 


\section{Applications}

In this section, we utilize our main theorem to prove a strong convergence theorem for finding a common element of the set of fixed points of a $\kappa$-quasi-strictly pseudocontractive mapping and the set of solutions of a finite family of variational inclusion problems and the set of solutions of a finite family of equilibrium problem in Hilbert space. To obtain this result, we recall some definitions, lemmas, and remarks as follows.

Definition 4.1 Let $C$ be a subset of a real Hilbert space $H$ and let $\mathcal{S}: C \rightarrow C$ be a mapping. Then $\mathcal{S}$ is said to be $\kappa$-quasi-strictly pseudo-contractive if there exists a constant $\kappa \in[0,1)$ such that

$$
\|\mathcal{S} u-p\|^{2} \leq\|u-p\|^{2}+\kappa\|u-\mathcal{S} u\|^{2}, \quad \forall u \in C \text { and } \forall p \in \operatorname{Fix}(\mathcal{S})
$$

$\mathcal{S}$ is said to be quasi-nonexpansive if

$$
\|\mathcal{S} u-p\| \leq\|u-p\|, \quad \forall u \in C \text { and } \forall p \in \operatorname{Fix}(\mathcal{S})
$$

The class of $\kappa$-quasi-strictly pseudo-contractions includes the class of quasi-nonexpansive mappings.

Remark 4.1 If $\mathcal{S}: C \rightarrow C$ be a $\kappa$-strictly pseudononspreading mapping with $\operatorname{Fix}(\mathcal{S}) \neq \emptyset$, then $\mathcal{S}$ is a $\kappa$-quasi-strictly pseudo-contractive mapping.

Example 4.2 Let $\mathcal{S}:[0,1] \rightarrow[0,1]$ be defined by

$$
\mathcal{S} u=\frac{2 u+1}{3}, \quad \text { for all } u \in[0,1]
$$

Then $\mathcal{S}$ is a $\kappa$-strictly pseudononspreading mapping where $\kappa \in[0,1)$. Since $1 \in \operatorname{Fix}(\mathcal{S}), \mathcal{S}$ is also $\kappa$-quasi-strictly pseudo-contractive mapping.

Next, we give the example to show that the converse of Remark 4.1 is not true.

Example 4.3 Let $\mathcal{S}:[-2,2] \rightarrow[-2,2]$ be defined by

$$
\mathcal{S} u=-\frac{5}{3} u, \quad \forall u \in[-2,2]
$$

First, show that $\mathcal{S}$ is a $\kappa$-quasi-strictly pseudo-contractive mapping for all $u \in[-2,2]$.

Observe that $\operatorname{Fix}(\mathcal{S})=\{0\}$. Let $u \in[-2,2]$, we have

$$
|\mathcal{S} u-\mathcal{S} 0|^{2}=\left|-\frac{5}{3} u-0\right|^{2}=\frac{25}{9}|u|^{2}
$$

and

$$
\begin{aligned}
|u-0|^{2}+\frac{1}{4}|(I-\mathcal{S}) u|^{2} & =|u|^{2}+\frac{1}{4}\left|u+\frac{5}{3} u\right|^{2} \\
& =|u|^{2}+\frac{1}{4}\left|\frac{8}{3} u\right|^{2}
\end{aligned}
$$




$$
\begin{aligned}
& =|u|^{2}+\frac{64}{9}\left(\frac{1}{4}\right)|u|^{2} \\
& =\left(\frac{25}{9}\right)|u|^{2} .
\end{aligned}
$$

Then $\mathcal{S}$ is a $\frac{1}{4}$-quasi-strictly pseudo-contractive mapping. Next, we show that $\mathcal{S}$ is not a $\frac{1}{4}$-strictly pseudononspreading mapping.

Choose $u=\frac{3}{2}$ and $v=\frac{-3}{2}$, we have

$$
\begin{aligned}
& \left|\mathcal{S}\left(\frac{3}{2}\right)-\mathcal{S}\left(-\frac{3}{2}\right)\right|^{2}=\left|-\frac{5}{3}\left(\frac{3}{2}\right)+\frac{5}{3}\left(-\frac{3}{2}\right)\right|^{2} \\
& =\left|-\frac{10}{2}\right|^{2} \\
& =25 \text {, } \\
& |u-v|^{2}=\left|\frac{3}{2}+\frac{3}{2}\right|^{2}=9 \\
& \frac{1}{4}\left|(I-\mathcal{S})\left(\frac{3}{2}\right)-(I-\mathcal{S})\left(\frac{-3}{2}\right)\right|^{2}=\frac{1}{4}\left|\left(\frac{3}{2}\right)+\frac{5}{3}\left(\frac{3}{2}\right)-\left(\left(-\frac{3}{2}\right)+\frac{5}{3}\left(-\frac{3}{2}\right)\right)\right|^{2} \\
& =\frac{1}{4}|8|^{2} \\
& =16
\end{aligned}
$$

and

$$
\begin{aligned}
2\left\langle(I-\mathcal{S})\left(\frac{3}{2}\right),(I-\mathcal{S})\left(\frac{-3}{2}\right)\right\rangle & =2\left\langle\left(\frac{3}{2}\right)+\frac{5}{3}\left(\frac{3}{2}\right),\left(\left(-\frac{3}{2}\right)+\frac{5}{3}\left(-\frac{3}{2}\right)\right)\right\rangle \\
& =2(4)(-4)=-32 .
\end{aligned}
$$

Then we have

$$
|\mathcal{S} u-\mathcal{S} v|^{2}>|u-v|^{2}+\frac{1}{4}|(I-\mathcal{S}) u-(I-\mathcal{S}) v|^{2}+2\langle u-\mathcal{S} u, v-\mathcal{S} v\rangle
$$

By changing $\mathcal{S}$ from being a $\kappa$-strictly pseudononspreading mapping with $\operatorname{Fix}(\mathcal{S}) \neq \varnothing$ into a $\kappa$-quasi-strictly pseudo-contractive mapping, we obtain the same result as in $\operatorname{Re}$ mark 2.10.

Remark 4.4 Let $\mathcal{S}: H \rightarrow H$ be a $\kappa$-quasi-strictly pseudo-contractive mapping with $\operatorname{Fix}(\mathcal{S}) \neq \emptyset$. Define $T: H \rightarrow H$ by $T u:=((1-\lambda) I+\lambda \mathcal{S}) u$, where $\lambda \in(0,1-\kappa)$. Then the following hold:

(i) $\operatorname{Fix}(\mathcal{S})=\operatorname{Fix}(T)=\operatorname{Fix}(I-\lambda(I-\mathcal{S}))$;

(ii) for every $u \in H$ and $v \in \operatorname{Fix}(\mathcal{S})$,

$$
\|T u-v\| \leq\|u-v\|
$$

In 2009, Kangtunyakarn and Suantai [16] introduced the $S$-mapping generated by $\mathcal{S}_{1}, \mathcal{S}_{2}$, $\ldots, \mathcal{S}_{N}$ and $\alpha_{1}, \alpha_{2}, \ldots, \alpha_{N}$ as follows. 
Definition 4.2 ([16]) Let $C$ be a nonempty convex subset of a real Banach space. Let $\left\{\mathcal{S}_{i}\right\}_{i=1}^{N}$ be a finite family of (nonexpansive) mappings of $C$ into itself. For each $j=1,2, \ldots$, let $\alpha_{j}=$ $\left(\alpha_{1}^{j}, \alpha_{2}^{j}, \alpha_{3}^{j}\right) \in I \times I \times I$ where $I=[0,1]$ and $\alpha_{1}^{j}+\alpha_{2}^{j}+\alpha_{3}^{j}=1$. Define the mapping $S: C \rightarrow C$ as follows:

$$
\begin{aligned}
& U_{0}=I, \\
& U_{1}=\alpha_{1}^{1} \mathcal{S}_{1} U_{0}+\alpha_{2}^{1} U_{0}+\alpha_{3}^{1} I, \\
& U_{2}=\alpha_{1}^{2} \mathcal{S}_{2} U_{1}+\alpha_{2}^{2} U_{1}+\alpha_{3}^{2} I, \\
& U_{3}=\alpha_{1}^{3} \mathcal{S}_{3} U_{2}+\alpha_{2}^{3} U_{2}+\alpha_{3}^{3} I, \\
& \vdots \\
& U_{n-1}=\alpha_{1}^{N-1} \mathcal{S}_{N-1} U_{N-2}+\alpha_{2}^{N-1} U_{N-2}+\alpha_{3}^{N-1} I, \\
& S=U_{n}=\alpha_{1}^{N} \mathcal{S}_{N} U_{n-1}+\alpha_{2}^{N} U_{n-1}+\alpha_{3}^{N} I .
\end{aligned}
$$

This mapping is called the $S$-mapping generated by $\mathcal{S}_{1}, \mathcal{S}_{2}, \ldots, \mathcal{S}_{N}$ and $\alpha_{1}, \alpha_{2}, \ldots, \alpha_{N}$.

Lemma 4.5 ([17]) Let $C$ be a nonempty closed convex subset of a real Hilbert space H. Let $\left\{\mathcal{S}_{i}\right\}_{i=1}^{N}$ be a finite family of nonspreading mappings of $C$ into itself with $\bigcap_{i=1}^{N} \operatorname{Fix}\left(\mathcal{S}_{i}\right) \neq \emptyset$ and let $\alpha_{j}=\left(\alpha_{1}^{j}, \alpha_{2}^{j}, \alpha_{3}^{j}\right) \in I \times I \times I$ where $I=[0,1], \alpha_{1}^{j}+\alpha_{2}^{j}+\alpha_{3}^{j}=1, \alpha_{1}^{j}, \alpha_{3}^{j} \in(0,1)$ for all $j=1,2, \ldots, N-1$ and $\alpha_{1}^{N} \in(0,1], \alpha_{3}^{N} \in[0,1), \alpha_{2}^{j} \in(0,1)$ for all $j=1,2, \ldots, N$. Let $S$ be the $S$-mapping generated by $\mathcal{S}_{1}, \mathcal{S}_{2}, \ldots, \mathcal{S}_{N}$ and $\alpha_{1}, \alpha_{2}, \ldots, \alpha_{N}$. Then $\operatorname{Fix}(S)=\bigcap_{i=1}^{N} \operatorname{Fix}\left(\mathcal{S}_{i}\right)$ and $S$ is a quasi-nonexpansive mapping.

Remark 4.6 From Lemma 4.5 it still holds if $C \equiv H$.

Theorem 4.7 Let $C$ be a nonempty closed convex subset of a real Hilbert space H. Let $M: H \rightarrow 2^{H}$ be a multi-valued maximal monotone mapping. For every $i=1,2, \ldots, N$, let $\Psi_{i}: C \times C \rightarrow \mathbb{R}$ be a bifunction satisfying (A1)-(A4) and $A_{i}: H \rightarrow H$ be $\alpha_{i}$-inverse strongly monotone mapping with $\eta=\min _{i=1,2, \ldots, N}\left\{\alpha_{i}\right\}$. Let $\mathcal{S}: H \rightarrow H$ be a $\kappa$-quasi-strictly pseudocontractive mapping. Assume $\Phi:=\operatorname{Fix}(\mathcal{S}) \cap \bigcap_{i=1}^{N} \operatorname{EP}\left(\Psi_{i}\right) \cap \bigcap_{i=1}^{N} \mathrm{VI}\left(H, A_{i}, M\right) \neq \emptyset$. Let the sequences $\left\{w_{n}\right\}$ and $\left\{z_{n}\right\}$ be generated by $w_{1}, \mu \in H$ and

$$
\left\{\begin{array}{c}
\sum_{i=1}^{N} a_{i} \Psi_{i}\left(z_{n}, y\right)+\frac{1}{r_{n}}\left\langle y-z_{n}, z_{n}-w_{n}\right\rangle \geq 0, \quad \forall y \in C, \\
w_{n+1}=\alpha_{n} \mu+\beta_{n} w_{n}+\gamma_{n} J_{M, \lambda}\left(I-\lambda \sum_{i=1}^{N} b_{i} A_{i}\right) w_{n} \\
+\eta_{n}\left(I-\rho_{n}(I-\mathcal{S})\right) w_{n}+\delta_{n} z_{n}, \quad \forall n \geq 1,
\end{array}\right.
$$

where $\left\{\alpha_{n}\right\},\left\{\beta_{n}\right\},\left\{\gamma_{n}\right\},\left\{\eta_{n}\right\},\left\{\delta_{n}\right\} \subseteq(0,1)$, and $\lambda>0$ with $\alpha_{n}+\beta_{n}+\gamma_{n}+\eta_{n}+\delta_{n}=1,0<\alpha<1$ and $0 \leq a_{i}, b_{i} \leq 1$, for every $i=1,2, \ldots, N, r_{n} \in[c, d] \subset(0,1), 0<p \leq \beta_{n}, \gamma_{n}, \eta_{n}, \delta_{n} \leq q<1$, $\rho_{n} \in(0,1-\kappa)$ for all $n \geq 1$. Suppose the following conditions hold:

(i) $\lim _{n \rightarrow \infty} \alpha_{n}=0$ and $\sum_{n=1}^{\infty} \alpha_{n}=\infty$,

(ii) $\sum_{n=1}^{\infty} \rho_{n}<\infty$,

(iii) $0<\lambda<2 \eta$, where $\eta=\min _{i=1,2, \ldots, N}\left\{\alpha_{i}\right\}$,

(iv) $\sum_{i=1}^{N} a_{i}=\sum_{i=1}^{N} b_{i}=1$,

(v) $\sum_{n=1}^{\infty}\left|\alpha_{n+1}-\alpha_{n}\right|<\infty, \sum_{n=1}^{\infty}\left|\beta_{n+1}-\beta_{n}\right|<\infty, \sum_{n=1}^{\infty}\left|\gamma_{n+1}-\gamma_{n}\right|<\infty$, $\sum_{n=1}^{\infty}\left|\rho_{n+1}-\rho_{n}\right|<\infty, \sum_{n=1}^{\infty}\left|\delta_{n+1}-\delta_{n}\right|<\infty, \sum_{n=1}^{\infty}\left|r_{n+1}-r_{n}\right|<\infty$.

Then the sequences $\left\{w_{n}\right\}$ and $\left\{z_{n}\right\}$ converge strongly to $\omega=P_{\Phi} \mu$. 
Proof Using Remark 4.4 and the same method of proof in Theorem 3.1, we have the desired conclusion.

Theorem 4.8 Let $C$ be a nonempty closed convex subset of a real Hilbert space $H$. Let $M: H \rightarrow 2^{H}$ be a multi-valued maximal monotone mapping. For every $i=1,2, \ldots, N$, let $\Psi_{i}: C \times C \rightarrow \mathbb{R}$ be a bifunction satisfying (A1)-(A4), and let $A_{i}: H \rightarrow H$ be $\alpha_{i}$-inverse strongly monotone mapping with $\eta=\min _{i=1,2, \ldots, N}\left\{\alpha_{i}\right\}$. Let $\mathcal{S}_{i}: H \rightarrow H$, for $i=1,2, \ldots, N$ be a finite family of nonspreading mappings with $\Psi:=\bigcap_{i=1}^{N} \operatorname{Fix}\left(\mathcal{S}_{i}\right) \cap \bigcap_{i=1}^{N} \operatorname{EP}\left(\Psi_{i}\right) \cap$ $\bigcap_{i=1}^{N} \operatorname{VI}\left(H, A_{i}, M\right) \neq \emptyset$. Let $\theta_{j}=\left(\alpha_{1}^{j}, \alpha_{2}^{j}, \alpha_{3}^{j}\right) \in I \times I \times I, j=1,2, \ldots, N$, where $I=[0,1]$, $\alpha_{1}^{j}+\alpha_{2}^{j}+\alpha_{3}^{j}=1, \alpha_{1}^{j}, \alpha_{3}^{j} \in(0,1)$ for all $j=1,2, \ldots, N-1$, and $\alpha_{1}^{N} \in(0,1], \alpha_{3}^{N} \in[0,1)$, $\alpha_{2}^{j} \in(0,1)$ for all $j=1,2, \ldots, N$, and let $S$ be the $S$-mapping generated by $\mathcal{S}_{1}, \mathcal{S}_{2}, \ldots, \mathcal{S}_{N}$ and $\theta_{1}, \theta_{2}, \ldots, \theta_{N}$. Let the sequences $\left\{w_{n}\right\}$ and $\left\{z_{n}\right\}$ be generated by $w_{1}, \mu \in H$ and

$$
\left\{\begin{array}{c}
\sum_{i=1}^{N} a_{i} \Psi_{i}\left(z_{n}, y\right)+\frac{1}{r_{n}}\left\langle y-z_{n}, z_{n}-w_{n}\right\rangle \geq 0, \quad \forall y \in C, \\
w_{n+1}=\alpha_{n} \mu+\beta_{n} w_{n}+\gamma_{n} J_{M, \lambda}\left(I-\lambda \sum_{i=1}^{N} b_{i} A_{i}\right) w_{n} \\
+\eta_{n}\left(I-\rho_{n}(I-S)\right) w_{n}+\delta_{n} z_{n}, \quad \forall n \geq 1
\end{array}\right.
$$

where $\left\{\alpha_{n}\right\},\left\{\beta_{n}\right\},\left\{\gamma_{n}\right\},\left\{\eta_{n}\right\},\left\{\delta_{n}\right\} \subseteq(0,1)$ and $\lambda>0$ with $\alpha_{n}+\beta_{n}+\gamma_{n}+\eta_{n}+\delta_{n}=1,0<\alpha<1$ and $0 \leq a_{i}, b_{i} \leq 1$, for every $i=1,2, \ldots, N, r_{n} \in[c, d] \subset(0,1), 0<p \leq \beta_{n}, \gamma_{n}, \eta_{n}, \delta_{n} \leq q<1$, $\rho_{n} \in(0,1)$ for all $n \geq 1$. Suppose the following conditions hold:

(i) $\lim _{n \rightarrow \infty} \alpha_{n}=0$ and $\sum_{n=1}^{\infty} \alpha_{n}=\infty$,

(ii) $\sum_{n=1}^{\infty} \rho_{n}<\infty$,

(iii) $0<\lambda<2 \eta$, where $\eta=\min _{i=1,2, \ldots, N}\left\{\alpha_{i}\right\}$,

(iv) $\sum_{i=1}^{N} a_{i}=\sum_{i=1}^{N} b_{i}=1$,

(v) $\sum_{n=1}^{\infty}\left|\alpha_{n+1}-\alpha_{n}\right|<\infty, \sum_{n=1}^{\infty}\left|\beta_{n+1}-\beta_{n}\right|<\infty, \sum_{n=1}^{\infty}\left|\gamma_{n+1}-\gamma_{n}\right|<\infty$, $\sum_{n=1}^{\infty}\left|\rho_{n+1}-\rho_{n}\right|<\infty, \sum_{n=1}^{\infty}\left|\delta_{n+1}-\delta_{n}\right|<\infty, \sum_{n=1}^{\infty}\left|r_{n+1}-r_{n}\right|<\infty$.

Then the sequences $\left\{w_{n}\right\}$ and $\left\{z_{n}\right\}$ converge strongly to $\omega=P_{\Phi} \mu$.

Proof From Theorem 4.7 and Remark 4.6, we obtain the desired conclusion.

\section{Numerical results}

The purpose of this section we give a numerical example to support our some result. The following example is given for supporting Theorem 3.1.

Example 5.1 Let $\mathbb{R}$ be the set of real numbers. For every $i=1,2, \ldots, N$, let $\Psi_{i}: \mathbb{R} \times \mathbb{R} \rightarrow \mathbb{R}$, $A_{i}: \mathbb{R} \rightarrow \mathbb{R}$ be defined by

$$
\begin{aligned}
& \Psi_{i}(u, v)=i(v-u)(3 u+v), \\
& A_{i} u=\frac{i u}{10}
\end{aligned}
$$

for all $u, v \in \mathbb{R}$ and let $\mathcal{S}: \mathbb{R} \rightarrow \mathbb{R}$ be defined by

$$
\mathcal{S} u= \begin{cases}\frac{-3 u}{5} & \text { if } u \in[0, \infty), \\ u & \text { if } u \in(-\infty, 0) .\end{cases}
$$


For every $i=1,2, \ldots, N$, suppose that $J_{M, \lambda}=I, \lambda=\frac{1}{N}, a_{i}=\frac{3}{4^{i}}+\frac{1}{N 4^{N}}, b_{i}=\frac{8}{9^{i}}+\frac{1}{N 9^{N}}$. Let $\left\{w_{n}\right\}$ and $\left\{z_{n}\right\}$ be generated by (3.1), where $\alpha_{n}=\frac{1}{20 n}, \beta_{n}=\frac{3(20 n-1)}{220 n}, \gamma_{n}=\frac{2(20 n-1)}{220 n}, \eta_{n}=\frac{5(20 n-1)}{220 n}, \delta_{n}=$ $\frac{20 n-1}{220 n}, r_{n}=\frac{3 n}{5 n+6}$, and $\rho_{n}=\frac{1}{2 n^{2}}$ for every $n \in \mathbb{N}$. Then the sequences $\left\{w_{n}\right\}$ and $\left\{z_{n}\right\}$ converge strongly to 0 .

Solution. It is easy to see that $\mathcal{S}$ is a $\kappa$-strictly pseudononspreading mapping. Since $a_{i}=$ $\frac{3}{4^{i}}+\frac{1}{N 4^{N}}$, we obtain

$$
\sum_{i=1}^{N} a_{i} \Psi_{i}(u, v)=\sum_{i=1}^{N}\left(\frac{3}{4^{i}}+\frac{1}{N 4^{N}}\right) i(v-u)(v+3 u) .
$$

It is easy to check that $\Psi_{i}$ satisfies all the conditions of Theorem 3.1 and $\operatorname{EP}\left(\sum_{i=1}^{N} a_{i} \Psi_{i}\right)=$ $\bigcap_{i=1}^{N} \mathrm{EP}\left(\Psi_{i}\right)=\{0\}$. Then we have

$$
\operatorname{Fix}(\mathcal{S}) \cap \bigcap_{i=1}^{N} \operatorname{EP}\left(\Psi_{i}\right)=\{0\} .
$$

Put $S_{1}=\sum_{i=1}^{N}\left(\frac{3}{4^{i}}+\frac{1}{N 4^{N}}\right) i$, then we have

$$
\begin{aligned}
0 & \leq \sum_{i=1}^{N} a_{i} \Psi_{i}\left(z_{n}, y\right)+\frac{1}{r_{n}}\left\langle y-z_{n}, z_{n}-w_{n}\right\rangle \\
= & S_{1}\left(y-z_{n}\right)\left(y+3 z_{n}\right)+\frac{1}{r_{n}}\left(y-z_{n}\right)\left(z_{n}-w_{n}\right) \\
\Leftrightarrow \quad 0 & \leq S_{1} r_{n}\left(y-z_{n}\right)\left(y+3 z_{n}\right)+\left(y-z_{n}\right)\left(z_{n}-w_{n}\right) \\
& =S_{1} r_{n} y^{2}+\left(z_{n}+2 r_{n} S_{1} z_{n}-w_{n}\right) y+z_{n} w_{n}-3 r_{n} S_{1} z_{n}^{2}-z_{n}^{2} .
\end{aligned}
$$

Let $G(y)=S_{1} r_{n} y^{2}+\left(z_{n}+2 r_{n} S_{1} z_{n}-w_{n}\right) y+z_{n} w_{n}-3 r_{n} S_{1} z_{n}^{2}-z_{n}^{2}$. $G(y)$ is a quadratic function of $y$ with coefficient $a=S_{1} r_{n}, b=z_{n}+2 r_{n} S_{1} z_{n}-w_{n}$, and $c=z_{n} w_{n}-3 r_{n} S_{1} z_{n}^{2}-z_{n}^{2}$. Determine the discriminant $\Delta$ of $G$ as follows:

$$
\begin{aligned}
\Delta & =b^{2}-4 a c \\
& =\left(z_{n}+2 r_{n} S_{1} z_{n}-w_{n}\right)^{2}-4\left(S_{1} r_{n}\right)\left(z_{n} w_{n}-3 r_{n} S_{1} z_{n}^{2}-z_{n}^{2}\right) \\
& =z_{n}^{2}+8 r_{n} S_{1} z_{n}^{2}+16 r_{n}^{2} S_{1}^{2} z_{n}^{2}-2 z_{n} w_{n}-8 r_{n} S_{1} z_{n} w_{n}+w_{n}^{2} \\
& =\left(z_{n}+4 S_{1} r_{n} z_{n}-w_{n}\right)^{2} .
\end{aligned}
$$

We know that $G(y) \geq 0, \forall y \in \mathbb{R}$. If it has at most one solution in $\mathbb{R}$, then $\Delta \leq 0$, so we obtain

$$
z_{n}=\frac{w_{n}}{1+4 S_{1} r_{n}}
$$

where $S_{1}=\sum_{i=1}^{N}\left(\frac{3}{4^{i}}+\frac{1}{N 4^{N}}\right) i$.

Since $A_{i} u=\frac{i u}{10}$ and $b_{i}=\frac{8}{9^{i}}+\frac{1}{N 9^{N}}$,

$$
\sum_{i=1}^{N} b_{i} A_{i} u=\sum_{i=1}^{N}\left(\frac{8}{9^{i}}+\frac{1}{N 9^{N}}\right) \frac{i u}{10} .
$$


Table 1 The values of the sequences $\left\{z_{n}\right\}$ and $\left\{w_{n}\right\}$ with initial values $\mu=w_{1}=5$

\begin{tabular}{|c|c|c|c|c|}
\hline \multirow[t]{2}{*}{$n$} & \multicolumn{2}{|l|}{$N=1$} & \multicolumn{2}{|l|}{$N=100$} \\
\hline & $\overline{z_{n}}$ & $w_{n}$ & $z_{n}$ & $w_{n}$ \\
\hline 1 & 2.391304 & 5.000000 & 2.037037 & 5.000000 \\
\hline 2 & 1.480316 & 3.700791 & 1.211068 & 3.633203 \\
\hline 3 & 0.982649 & 2.667191 & 0.784335 & 2.577101 \\
\hline 4 & 0.667619 & 1.900147 & 0.523043 & 1.810533 \\
\hline 5 & 0.459689 & 1.349410 & 0.354712 & 1.270096 \\
\hline : & $\vdots$ & 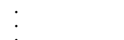 & & \\
\hline 50 & 0.004726 & 0.015803 & 0.003739 & 0.015422 \\
\hline : & $\vdots$ & $\vdots$ & $\vdots$ & : \\
\hline 96 & 0.002359 & 0.007951 & 0.001867 & 0.007854 \\
\hline 97 & 0.002334 & 0.007866 & 0.001847 & 0.007687 \\
\hline 98 & 0.002309 & 0.007783 & 0.001828 & 0.007606 \\
\hline 99 & 0.002284 & 0.007702 & 0.001808 & 0.007526 \\
\hline 100 & 0.002261 & 0.007622 & 0.001790 & 0.007448 \\
\hline
\end{tabular}

From (5.1) and the definition of $A_{i}$, we have

$$
\operatorname{Fix}(\mathcal{S}) \cap \bigcap_{i=1}^{N} \operatorname{EP}\left(\Psi_{i}\right) \cap \bigcap_{i=1}^{N} \operatorname{VI}\left(H, A_{i}, M\right)=\{0\}
$$

For every $n \in \mathbb{N}, \alpha_{n}=\frac{1}{20 n}, \beta_{n}=\frac{3(20 n-1)}{220 n}, \gamma_{n}=\frac{2(20 n-1)}{220 n}, \eta_{n}=\frac{20 n-1}{220 n}, \delta_{n}=\frac{5(20 n-1)}{220 n}, r_{n}=\frac{3 n}{5 n+6}$, and $\rho_{n}=\frac{1}{2 n^{2}}$. Then the sequences $\left\{\alpha_{n}\right\},\left\{\beta_{n}\right\},\left\{\gamma_{n}\right\},\left\{\eta_{n}\right\},\left\{\delta_{n}\right\},\left\{r_{n}\right\}$, and $\left\{\rho_{n}\right\}$ satisfy all the conditions of Theorem 3.1. For every $n \in \mathbb{N}$, from (5.2), we rewrite (3.1) as follows:

$$
\begin{aligned}
w_{n+1}= & \frac{1}{20 n} \mu+\frac{3(20 n-1)}{220 n} w_{n}+\frac{2(20 n-1)}{220 n}\left(w_{n}-\frac{1}{N} \sum_{i=1}^{N}\left(\frac{8}{9^{i}}+\frac{1}{N 9^{N}}\right) \frac{i w_{n}}{10}\right) \\
& +\frac{5(20 n-1)}{220 n}\left(I-\frac{1}{2 n^{2}}(I-\mathcal{S})\right) w_{n} \\
& +\frac{20 n-1}{220 n}\left(\frac{w_{n}}{1+4\left(\sum_{i=1}^{N}\left(\frac{3}{4^{i}}+\frac{1}{N 4^{N}}\right) i\right) \frac{3 n}{5 n+6}}\right) .
\end{aligned}
$$

Using the algorithm (5.4) and choosing $\mu=w_{1}=5$ with $N=1$ and $N=100$, we have the numerical results in Table 1.

\section{Conclusion}

1. The sequences $\left\{w_{n}\right\}$ and $\left\{z_{n}\right\}$ converge to 0 as shown in Table 1 and Figure 1.

2. From Theorem 3.1, we can conclude that the sequences $\left\{w_{n}\right\}$ and $\left\{z_{n}\right\}$, in

Example 5.1, converge to 0 .

Next, we give the numerical example to support our some result in a three dimensional space of real numbers.

Example 5.2 Let an inner product $\langle\cdot, \cdot\rangle: \mathbb{R}^{3} \times \mathbb{R}^{3} \rightarrow \mathbb{R}$ be defined by $\langle\mathbf{w}, \mathbf{y}\rangle=\mathbf{w} \cdot \mathbf{y}=w_{1}$. $y_{1}+w_{2} \cdot y_{2}+w_{3} \cdot y_{3}$ and a usual norm $\|\cdot\|: \mathbb{R}^{3} \rightarrow \mathbb{R}$ defined by $\|\mathbf{w}\|=\sqrt{w_{1}^{2}+w_{2}^{2}+w_{3}^{2}}$ for all $\mathbf{w}=\left(w_{1}, w_{2}, w_{3}\right), \mathbf{y}=\left(y_{1}, y_{2}, y_{3}\right) \in \mathbb{R}^{3}$. For every $i=1,2, \ldots, N$, let $\Psi_{i}: \mathbb{R}^{3} \times \mathbb{R}^{3} \rightarrow \mathbb{R}$, $A_{i}: \mathbb{R}^{3} \rightarrow \mathbb{R}^{3}$ be defined by

$$
\Psi_{i}(\mathbf{w}, \mathbf{y})=i(\mathbf{y}-\mathbf{w}) \cdot(9 \mathbf{w}+\mathbf{y}), \quad A_{i} \mathbf{w}=\left(\frac{i w_{1}}{6}, \frac{i w_{2}}{6}, \frac{i w_{3}}{6}\right)
$$




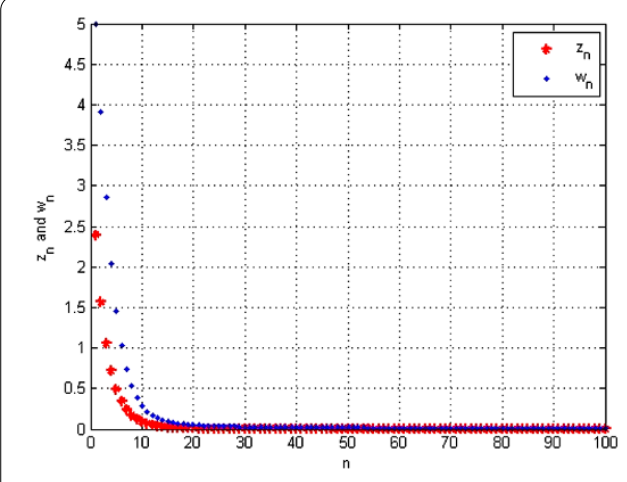

(a) $N=1$

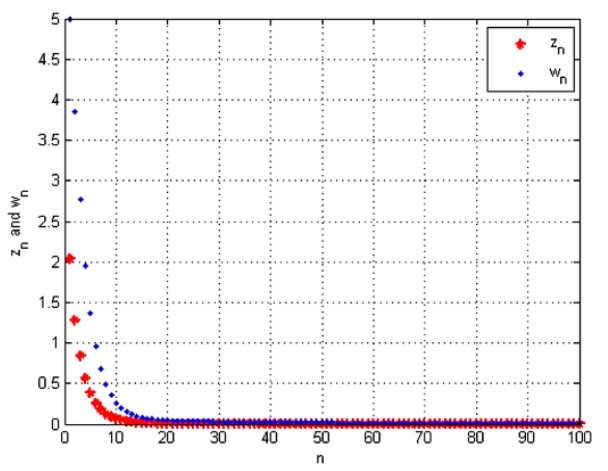

(b) $N=100$

Figure 1 The convergence of $\left\{z_{n}\right\}$ and $\left\{w_{n}\right\}$ with initial values $\mu=w_{1}=5$.

for all $\mathbf{w}=\left(w_{1}, w_{2}, w_{3}\right) \in \mathbb{R}^{3}, \mathbf{y}=\left(y_{1}, y_{2}, y_{3}\right) \in \mathbb{R}^{3}$ and let $\mathcal{S}: \mathbb{R}^{3} \rightarrow \mathbb{R}^{3}$ be defined by

$$
\mathcal{S} \mathbf{w}= \begin{cases}\left(\frac{-3 w_{1}}{5}, \frac{-5 w_{2}}{7}, \frac{-7 w_{3}}{9}\right) & \text { if } w_{i} \in[0, \infty) \text { for all } i=1,2,3 \\ \left(w_{1}, w_{2}, w_{3}\right) & \text { if } w_{i} \in(-\infty, 0) \text { for all } i=1,2,3\end{cases}
$$

For every $i=1,2, \ldots, N$, suppose that $J_{M, \lambda}=I, \lambda=\frac{1}{N}, a_{i}=\frac{4}{5^{i}}+\frac{1}{N 5^{N}}, b_{i}=\frac{7}{8^{i}}+\frac{1}{N 8^{N}}$. Let $\mathbf{w}_{n}=\left(w_{n}^{1}, w_{n}^{2}, w_{n}^{3}\right)$ and $\mathbf{z}_{n}=\left(z_{n}^{1}, z_{n}^{2}, z_{n}^{3}\right)$ be generated by (3.1), where $\alpha_{n}=\frac{1}{15 n}, \beta_{n}=\frac{3(15 n-1)}{165 n}$, $\gamma_{n}=\frac{2(15 n-1)}{165 n}, \eta_{n}=\frac{5(15 n-1)}{165 n}, \delta_{n}=\frac{15 n-1}{165 n}, r_{n}=\frac{2 n}{7 n+6}$, and $\rho_{n}=\frac{1}{9 n^{2}}$ for every $n \in \mathbb{N}$. Then the sequences $\mathbf{w}_{n}=\left(w_{n}^{1}, w_{n}^{2}, w_{n}^{3}\right)$ and $\mathbf{z}_{n}=\left(z_{n}^{1}, z_{n}^{2}, z_{n}^{3}\right)$ converge strongly to $\mathbf{0}$ where $\mathbf{0}=(0,0,0)$.

Solution. It is easy to see that $\mathcal{S}$ is a $\frac{7}{8}$-strictly pseudononspreading mapping. Since $a_{i}=$ $\frac{3}{4^{i}}+\frac{1}{N 4^{N}}$, we obtain

$$
\sum_{i=1}^{N} a_{i} \Psi_{i}(\mathbf{w}, \mathbf{y})=\sum_{i=1}^{N}\left(\frac{4}{5^{i}}+\frac{1}{N 5^{N}}\right) i(\mathbf{y}-\mathbf{w}) \cdot(\mathbf{y}+9 \mathbf{w}),
$$

for all $\mathbf{w}=\left(w_{1}, w_{2}, w_{3}\right) \in \mathbb{R}^{3}, \mathbf{y}=\left(y_{1}, y_{2}, y_{3}\right) \in \mathbb{R}^{3}$. It is easy to check that $\Psi_{i}$ satisfies the condition of Theorem 3.1 and $\operatorname{EP}\left(\sum_{i=1}^{N} a_{i} \Psi_{i}\right)=\bigcap_{i=1}^{N} \operatorname{EP}\left(\Psi_{i}\right)=\{\mathbf{0}\}$. Then we have

$$
\operatorname{Fix}(\mathcal{S}) \cap \bigcap_{i=1}^{N} \operatorname{EP}\left(\Psi_{i}\right)=\{\mathbf{0}\} .
$$

Put $S_{2}=\sum_{i=1}^{N}\left(\frac{4}{5^{i}}+\frac{1}{N 5^{N}}\right) i$, we have

$$
\begin{aligned}
0 \leq & \sum_{i=1}^{N} a_{i} \Psi_{i}\left(\mathbf{z}_{n}, \mathbf{y}\right)+\frac{1}{r_{n}}\left\langle\mathbf{y}-\mathbf{z}_{n}, \mathbf{z}_{n}-\mathbf{w}_{n}\right\rangle \\
= & S_{2}\left(\mathbf{y}-\mathbf{z}_{n}\right) \cdot\left(\mathbf{y}+9 \mathbf{z}_{n}\right)+\frac{1}{r_{n}}\left(\mathbf{y}-\mathbf{z}_{n}\right)\left(\mathbf{z}_{n}-\mathbf{w}_{n}\right) \\
= & S_{2}\left(y_{1}-z_{n}^{1}, y_{2}-z_{n}^{2}, y_{3}-z_{n}^{3}\right) \cdot\left(y_{1}+9 z_{n}^{1}, y_{2}+9 z_{n}^{2}, y_{3}+9 z_{n}^{3}\right) \\
& +\frac{1}{r_{n}}\left(y_{1}-z_{n}^{1}, y_{2}-z_{n}^{2}, y_{3}-z_{n}^{3}\right) \cdot\left(z_{n}^{1}-w_{n}^{1}, z_{n}^{2}-w_{n}^{2}, z_{n}^{3}-w_{n}^{3}\right) \\
= & S_{2}\left(\left(y_{1}-z_{n}^{1}\right)\left(y_{1}+9 z_{n}^{1}\right)+\left(y_{2}-z_{n}^{2}\right)\left(y_{2}+9 z_{n}^{2}\right)+\left(y_{3}-z_{n}^{3}\right)\left(y_{3}+9 z_{n}^{3}\right)\right)
\end{aligned}
$$




$$
\begin{aligned}
&+ \frac{1}{r_{n}}\left(\left(y_{1}-z_{n}^{1}\right)\left(z_{n}^{1}-w_{n}^{1}\right)+\left(y_{2}-z_{n}^{2}\right)\left(z_{n}^{2}-w_{n}^{2}\right)+\left(y_{3}-z_{n}^{3}\right)\left(z_{n}^{3}-w_{n}^{3}\right)\right) \\
&=\left(S_{2}\left(y_{1}-z_{n}^{1}\right)\left(y_{1}+9 z_{n}^{1}\right)+\frac{1}{r_{n}}\left(y_{1}-z_{n}^{1}\right)\left(z_{n}^{1}-w_{n}^{1}\right)\right)+\left(S_{2}\left(y_{2}-z_{n}^{2}\right)\left(y_{2}+9 z_{n}^{2}\right)+\frac{1}{r_{n}}\left(y_{2}-z_{n}^{2}\right)\left(z_{n}^{2}-w_{n}^{2}\right)\right) \\
&+\left(S_{2}\left(y_{3}-z_{n}^{3}\right)\left(y_{3}+9 z_{n}^{3}\right)+\frac{1}{r_{n}}\left(y_{3}-z_{n}^{3}\right)\left(z_{n}^{3}-w_{n}^{3}\right)\right) \\
& \Leftrightarrow \quad 0 \leq\left(S_{2} r_{n}\left(y_{1}-z_{n}^{1}\right)\left(y_{1}+9 z_{n}^{1}\right)+\left(y_{1}-z_{n}^{1}\right)\left(z_{n}^{1}-w_{n}^{1}\right)\right) \\
&+\left(S_{2} r_{n}\left(y_{2}-z_{n}^{2}\right)\left(y_{2}+9 z_{n}^{2}\right)+\left(y_{2}-z_{n}^{2}\right)\left(z_{n}^{2}-w_{n}^{2}\right)\right) \\
&+\left(S_{2} r_{n}\left(y_{3}-z_{n}^{3}\right)\left(y_{3}+9 z_{n}^{3}\right)+\left(y_{3}-z_{n}^{3}\right)\left(z_{n}^{3}-w_{n}^{3}\right)\right) \\
&=\left(S_{2} r_{n}\left(y_{1}\right)^{2}+\left(z_{n}^{1}+8 r_{n} S_{2} z_{n}^{1}-w_{n}^{1}\right) y_{1}+z_{n}^{1} w_{n}^{1}-9 r_{n} S_{2}\left(z_{n}^{1}\right)^{2}-\left(z_{n}^{1}\right)^{2}\right) \\
&+\left(S_{2} r_{n}\left(y_{2}\right)^{2}+\left(z_{n}^{2}+8 r_{n} S_{2} z_{n}^{2}-w_{n}^{2}\right) y_{2}+z_{n}^{2} w_{n}^{2}-9 r_{n} S_{2}\left(z_{n}^{2}\right)^{2}-\left(z_{n}^{2}\right)^{2}\right) \\
&+\left(S_{2} r_{n}\left(y_{3}\right)^{2}+\left(z_{n}^{3}+8 r_{n} S_{2} z_{n}^{3}-w_{n}^{3}\right) y_{3}+z_{n}^{3} w_{n}^{3}-9 r_{n} S_{2}\left(z_{n}^{3}\right)^{2}-\left(z_{n}^{3}\right)^{2}\right) \\
&= G\left(y_{1}\right)+G\left(y_{2}\right)+G\left(y_{3}\right),
\end{aligned}
$$

where $G\left(y_{1}\right)=S_{2} r_{n}\left(y_{1}\right)^{2}+\left(z_{n}^{1}+8 r_{n} S_{2} z_{n}^{1}-w_{n}^{1}\right) y_{1}+z_{n}^{1} w_{n}^{1}-9 r_{n} S_{2}\left(z_{n}^{1}\right)^{2}-\left(z_{n}^{1}\right)^{2}, G\left(y_{2}\right)=S_{2} r_{n}\left(y_{2}\right)^{2}+$ $\left(z_{n}^{2}+8 r_{n} S_{2} z_{n}^{2}-w_{n}^{2}\right) y_{2}+z_{n}^{2} w_{n}^{2}-9 r_{n} S_{2}\left(z_{n}^{2}\right)^{2}-\left(z_{n}^{2}\right)^{2}$ and $G\left(y_{3}\right)=S_{2} r_{n}\left(y_{3}\right)^{2}+\left(z_{n}^{3}+8 r_{n} S_{2} z_{n}^{3}-\right.$ $\left.w_{n}^{3}\right) y_{3}+z_{n}^{3} w_{n}^{3}-9 r_{n} S_{2}\left(z_{n}^{3}\right)^{2}-\left(z_{n}^{3}\right)^{2}$. Then $G\left(y_{1}\right), G\left(y_{2}\right)$, and $G\left(y_{3}\right)$ are a quadratic function of y with coefficients $a_{1}=S_{2} r_{n}, b_{1}=z_{n}^{1}+8 r_{n} S_{2} z_{n}^{1}-w_{n}^{1}, c_{1}=z_{n}^{1} w_{n}^{1}-9 r_{n} S_{2}\left(z_{n}^{1}\right)^{2}-\left(z_{n}^{1}\right)^{2}, a_{2}=S_{2} r_{n}$, $b_{2}=z_{n}^{2}+8 r_{n} S_{2} z_{n}^{2}-w_{n}^{2}, c_{2}=z_{n}^{2} w_{n}^{2}-9 r_{n} S_{2}\left(z_{n}^{2}\right)^{2}-\left(z_{n}^{2}\right)^{2}, a_{3}=S_{2} r_{n}, b_{3}=z_{n}^{3}+8 r_{n} S_{2} z_{n}^{3}-w_{n}^{3}$ and $c_{3}=z_{n}^{3} w_{n}^{3}-9 r_{n} S_{2}\left(z_{n}^{3}\right)^{2}-\left(z_{n}^{3}\right)^{2}$, respectively. Determine the discriminant $\Delta_{1}$ of $G_{1}$ as follows:

$$
\begin{aligned}
\Delta_{1} & =b_{1}^{2}-4 a_{1} c_{1} \\
& =\left(z_{n}^{1}+8 r_{n} S_{2} z_{n}^{1}-w_{n}^{1}\right)^{2}-4\left(S_{2} r_{n}\right)\left(z_{n}^{1} w_{n}^{1}-9 r_{n} S_{2}\left(z_{n}^{1}\right)^{2}-\left(z_{n}^{1}\right)^{2}\right) \\
& =\left(z_{n}^{1}\right)^{2}+20 r_{n} S_{2}\left(z_{n}^{1}\right)^{2}+100 r_{n}^{2} S_{2}^{2}\left(z_{n}^{1}\right)^{2}-2 z_{n}^{1} w_{n}^{1}-20 r_{n} S_{2} z_{n}^{1} w_{n}^{1}+\left(w_{n}^{1}\right)^{2} \\
& =\left(z_{n}^{1}+10 S_{2} r_{n} z_{n}^{1}-w_{n}^{1}\right)^{2} .
\end{aligned}
$$

From (5.6), if $G\left(y_{1}\right) \geq 0, \forall y_{1} \in \mathbb{R}$ and it has most one solution in $\mathbb{R}$, then $\Delta_{1} \leq 0$, so we obtain

$$
z_{n}^{1}=\frac{w_{n}^{1}}{1+10 S_{2} r_{n}} .
$$

Next, determine the discriminant $\Delta_{2}$ of $G_{2}$ as follows:

$$
\begin{aligned}
\Delta_{2} & =b_{2}^{2}-4 a_{2} c_{2} \\
& =\left(z_{n}^{2}+8 r_{n} S_{2} z_{n}^{2}-w_{n}^{2}\right)^{2}-4\left(S_{2} r_{n}\right)\left(z_{n}^{2} w_{n}^{2}-9 r_{n} S_{2}\left(z_{n}^{2}\right)^{2}-\left(z_{n}^{2}\right)^{2}\right) \\
& =\left(z_{n}^{2}\right)^{2}+20 r_{n} S_{2}\left(z_{n}^{2}\right)^{2}+100 r_{n}^{2} S_{2}^{2}\left(z_{n}^{2}\right)^{2}-2 z_{n}^{2} w_{n}^{2}-20 r_{n} S_{2} z_{n}^{2} w_{n}^{2}+\left(w_{n}^{2}\right)^{2} \\
& =\left(z_{n}^{2}+10 S_{2} r_{n} z_{n}^{2}-w_{n}^{2}\right)^{2} .
\end{aligned}
$$


From (5.6), if $G\left(y_{2}\right) \geq 0, \forall y_{2} \in \mathbb{R}$ and it has at most one solution in $\mathbb{R}$, then $\Delta_{2} \leq 0$, so we obtain

$$
z_{n}^{2}=\frac{w_{n}^{2}}{1+10 S_{2} r_{n}}
$$

Next, determine the discriminant $\Delta_{3}$ of $G_{3}$ as follows:

$$
\begin{aligned}
\Delta_{3} & =b_{3}^{2}-4 a_{3} c_{3} \\
& =\left(z_{n}^{3}+8 r_{n} S_{2} z_{n}^{3}-w_{n}^{3}\right)^{2}-4\left(S_{2} r_{n}\right)\left(z_{n}^{3} w_{n}^{3}-9 r_{n} S_{2}\left(z_{n}^{3}\right)^{2}-\left(z_{n}^{3}\right)^{2}\right) \\
& =\left(z_{n}^{3}\right)^{2}+20 r_{n} S_{2}\left(z_{n}^{3}\right)^{2}+100 r_{n}^{2} S_{2}^{2}\left(z_{n}^{3}\right)^{2}-2 z_{n}^{3} w_{n}^{3}-20 r_{n} S_{2} z_{n}^{3} w_{n}^{3}+\left(w_{n}^{3}\right)^{2} \\
& =\left(z_{n}^{3}+10 S_{2} r_{n} z_{n}^{3}-w_{n}^{3}\right)^{2} .
\end{aligned}
$$

From (5.6), if $G\left(y_{3}\right) \geq 0, \forall y_{3} \in \mathbb{R}$ and it has at most one solution in $\mathbb{R}$, then $\Delta_{3} \leq 0$, so we obtain

$$
z_{n}^{3}=\frac{w_{n}^{3}}{1+10 S_{2} r_{n}}
$$

Since $A_{i} \mathbf{w}=\left(\frac{i w_{1}}{6}, \frac{i w_{2}}{6}, \frac{i w_{3}}{6}\right)$ and $b_{i}=\frac{7}{8^{i}}+\frac{1}{N 8^{N}}$, then

$$
\sum_{i=1}^{N} b_{i} A_{i} \mathbf{w}=\sum_{i=1}^{N}\left(\frac{7}{8^{i}}+\frac{1}{N 8^{N}}\right) A_{i} \mathbf{w}
$$

From (5.5) and the definition of $A_{i}$, we have

$$
\operatorname{Fix}(\mathcal{S}) \cap \bigcap_{i=1}^{N} \operatorname{EP}\left(\Psi_{i}\right) \cap \bigcap_{i=1}^{N} \operatorname{VI}\left(H, A_{i}, M\right)=\{\mathbf{0}\}
$$

For every $n \in \mathbb{N}, \alpha_{n}=\frac{1}{15 n}, \beta_{n}=\frac{3(15 n-1)}{165 n}, \gamma_{n}=\frac{2(15 n-1)}{165 n}, \eta_{n}=\frac{5(15 n-1)}{165 n}, \delta_{n}=\frac{15 n-1}{165 n}, r_{n}=\frac{2 n}{7 n+6}$, and $\rho_{n}=\frac{1}{9 n^{2}}$. Then the sequences $\left\{\alpha_{n}\right\},\left\{\beta_{n}\right\},\left\{\gamma_{n}\right\},\left\{\eta_{n}\right\},\left\{\delta_{n}\right\},\left\{r_{n}\right\}$, and $\left\{\rho_{n}\right\}$ satisfy all the conditions of Theorem 3.1. For every $n \in \mathbb{N}$, from (5.7), (5.8), and (5.9), we rewrite (3.1) as follows:

$$
\begin{aligned}
\mathbf{w}_{n+1}= & \frac{1}{15 n} \boldsymbol{\mu}+\frac{3(15 n-1)}{165 n} \mathbf{w}_{n}+\frac{2(15 n-1)}{165 n}\left(\mathbf{w}_{n}-\frac{1}{N} \sum_{i=1}^{N}\left(\frac{7}{8^{i}}+\frac{1}{N 8^{N}}\right) A_{i} \mathbf{w}_{n}\right) \\
& +\frac{5(15 n-1)}{165 n}\left(I-\frac{1}{2 n^{2}}(I-\mathcal{S})\right) \mathbf{w}_{n}+\frac{15 n-1}{165 n} \mathbf{z}_{n},
\end{aligned}
$$

where $\mathbf{w}_{n}=\left(w_{n}^{1}, w_{n}^{2}, w_{n}^{3}\right)$ and $\mathbf{z}_{n}=\left(z_{n}^{1}, z_{n}^{2}, z_{n}^{3}\right)=\left(\frac{w_{n}^{1}}{1+10 S_{2} r_{n}}, \frac{w_{n}^{2}}{1+10 S_{2} r_{n}}, \frac{w_{n}^{3}}{1+10 S_{2} r_{n}}\right)$.

Using the algorithm (5.11), choose $\mu=(5,10,15), \mathbf{w}_{1}=(2,12,20), n=100$, and $N=100$. The numerical results for the sequences $\mathbf{w}_{n}$ and $\mathbf{z}_{n}$ are shown in Table 2 and Figure 2.

\section{Conclusion}

1. The sequences $\left\{\mathbf{w}_{n}\right\}$ and $\left\{\mathbf{z}_{n}\right\}$ converge to $\mathbf{0}$ as shown in Table 2 and Figure 2 .

2. From Theorem 3.1, we can conclude that the sequences $\left\{\mathbf{w}_{n}\right\}$ and $\left\{\mathbf{z}_{n}\right\}$, in Example 5.2, converge to $\mathbf{0 .}$ 
Table 2 The values of the sequences $\left\{z_{n}\right\}$ and $\left\{w_{n}\right\}$ with initial values $\mu=(5,10,15)$, $\mathrm{w}_{1}=(2,12,20)$, and $n=N=100$

\begin{tabular}{rll}
\hline $\boldsymbol{n}$ & $\mathbf{z}_{\boldsymbol{n}}$ & $\mathbf{w}_{\boldsymbol{n}}$ \\
\hline 1 & $(0.453608,2.721649,4.536082)$ & $(2.000000,12.000000,20.000000)$ \\
2 & $(0.336995,1.776168,2.930220)$ & $(1.916656,10.101958,16.665628)$ \\
3 & $(0.289738,1.439132,2.364314)$ & $(1.841903,9.148767,15.030279)$ \\
4 & $(0.259512,1.239451,2.030799)$ & $(1.756695,8.390131,13.746949)$ \\
5 & $(0.236507,1.096332,1.792646)$ & $(1.666990,7.727376,12.635260)$ \\
$\vdots$ & $\vdots$ & $\vdots$ \\
50 & $(0.017724,0.045633,0.070850)$ & $(0.147537,0.379859,0.589773)$ \\
$\vdots$ & $\vdots$ & $\vdots$ \\
96 & $(0.006069,0.012338,0.018554)$ & $(0.051022,0.103729,0.155990)$ \\
97 & $(0.005987,0.012158,0.018280)$ & $(0.050339,0.102226,0.153703)$ \\
98 & $(0.005907,0.011984,0.018015)$ & $(0.049677,0.100775,0.151496)$ \\
99 & $(0.005830,0.011816,0.017760)$ & $(0.049033,0.099372,0.149365)$ \\
100 & $(0.005755,0.011653,0.017513)$ & $(0.048408,0.098015,0.147304)$ \\
\hline & &
\end{tabular}

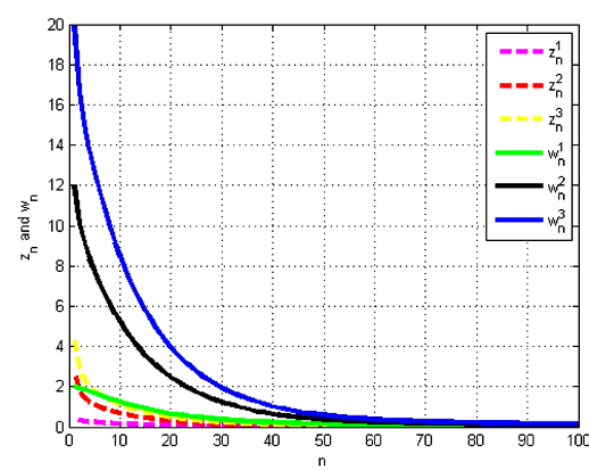

(a) $2 \mathrm{D}$

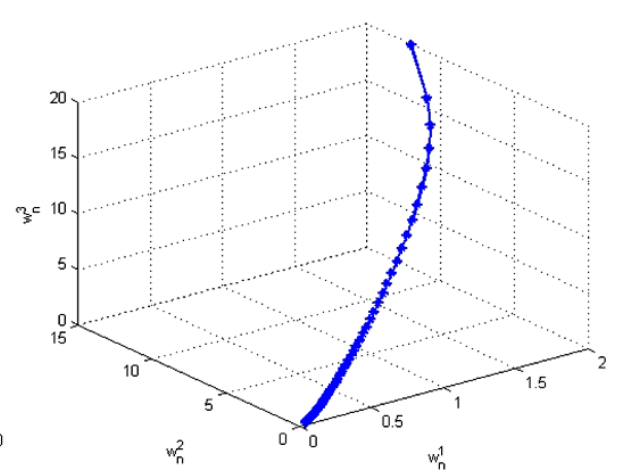

(b) $3 \mathrm{D}$

Figure 2 The convergence of $\left\{z_{n}\right\}$ and $\left\{w_{n}\right\}$ with initial values $\mu=(5,10,15), w_{1}=(2,12,20)$, and $n=N=100$.

\section{Competing interests}

The authors declare that they have no competing interests.

\section{Authors' contributions}

Both authors contributed equally and significantly to this research article. Both authors read and approved the final manuscript.

\section{Acknowledgements}

The authors appreciated the referees for providing valuable comments improving the content of this research paper. This research was supported by the Research Administration Division of King Mongkut's Institute of Technology Ladkrabang.

Received: 13 February 2014 Accepted: 15 September 2014 Published: 13 Oct 2014

\section{References}

1. Kohsaka, F, Takahashi, W: Fixed point theorems for a class of nonlinear mappings related to maximal monotone operators in Banach spaces. Arch. Math. 91, 166-177 (2008)

2. Iemoto, S, Takahashi, W: Approximating common fixed points of nonexpansive mappings and nonspreading mappings in a Hilbert space. Nonlinear Anal. 71, 2080-2089 (2009)

3. Osilike, MO, Isiogugu, FO: Weak and strong convergence theorems for nonspreading-type mappings in Hilbert spaces. Nonlinear Anal. 74, 1814-1822 (2011)

4. Kangtunyakarn, A: Iterative algorithms for finding a common solution of system of the set of variational inclusion problems and the set of fixed point problems. Fixed Point Theory Appl. 2011, 38 (2011)

5. Chang, SS: Set-valued variational inclusions in Banach spaces. J. Math. Anal. Appl. 248, 438-454 (2000)

6. Hao, Y: On variational inclusion and common fixed point problems in Hilbert spaces with applications. Appl. Math. Comput. 217(7), 3000-3010 (2010)

7. Zhang, SS, Lee, JHW, Chan, CK: Algorithms of common solutions for quasi-variational inclusion and fixed point problems. Appl. Math. Mech. 29, 571-581 (2008) 
8. Combettes, PL, Hirstoaga, SA: Equilibrium programming in Hilbert spaces. J. Nonlinear Convex Anal. 6(1), 117-136 (2005)

9. Takahashi, S, Takahashi, W: Viscosity approximation methods for equilibrium problems and fixed point problems in Hilbert spaces. J. Math. Anal. Appl. 331, 506-515 (2007)

10. Kangtunyakarn, $A$ : The methods for variational inequality problems and fixed point of $\boldsymbol{\kappa}_{i}$-strictly pseudononspreading mapping. Fixed Point Theory Appl. 2013, 171 (2013). doi:10.1186/1687-1812-2013-171

11. Suwannaut, S, Kangtunyakarn, A: The combination of the set of solutions of equilibrium problem for convergence theorem of the set of fixed points of strictly pseudo-contractive mappings and variational inequalities problem. Fixed Point Theory Appl. 2013, 291 (2013). doi:10.1186/1687-1812-2013-291

12. Takahashi, W: Nonlinear Functional Analysis. Yokohama Publishers, Yokohama (2000)

13. Xu, HK: An iterative approach to quadric optimization. J. Optim. Theory Appl. 116, 659-678 (2003)

14. Zegeye, H, Shahzad, N: Convergence of Mann's type iteration method for generalized asymptotically nonexpansive mappings. Comput. Math. Appl. 62, 4007-4014 (2011)

15. Blum, E, Oettli, W: From optimization and variational inequalities to equilibrium problems. Math. Stud. 63(14), 123-145 (1994)

16. Kangtunyakarn, A, Suantai, S: Hybrid iterative scheme for generalized equilibrium problems and fixed point problems of finite family of nonexpansive mappings. Nonlinear Anal. Hybrid Syst. 3, 296-309 (2009)

17. Kangtunyakarn, A: Strong convergence of the hybrid method for a finite family of nonspreading mappings and variational inequality problems. Fixed Point Theory Appl. 2012, 188 (2012)

10.1186/1687-1812-2014-209

Cite this article as: Khuangsatung and Kangtunyakarn: Algorithm of a new variational inclusion problem and strictly pseudononspreading mapping with application. Fixed Point Theory and Applications 2014, 2014:209

\section{Submit your manuscript to a SpringerOpen ${ }^{\circ}$ journal and benefit from:}

- Convenient online submission

- Rigorous peer review

- Immediate publication on acceptance

- Open access: articles freely available online

- High visibility within the field

- Retaining the copyright to your article 